\title{
LINEAR CONNEXIONS WITH ZERO TORSION AND RECURRENT CURVATURE
}

\author{
BY \\ YUNG-CHOW WONG
}

Introduction. Let $M$ be a connected $n$-dimensional $C^{\infty}$-manifold. If $S$ is any tensor (field) on $M$, then

(i) $S(u)$ denotes the value of $S$ at the point $u \in M$;

(ii) $S=0$ means that $S$ is everywhere zero, i.e. $S(u)=0$ for every $u \in M$;

(iii) $S \not \equiv 0$ means that $S$ is not everywhere zero, i.e. $S(u) \neq 0$ for some $u \in M$;

(iv) $S \neq 0$ means that $S$ is nowhere zero, i.e. $S(u)=0$ for no $u \in M$.

Throughout this paper, a tensor of type $(1,0)$ will be called a vector, and a tensor of type $(0,1)$ a covector. Summation over a repeated index, Latin or Greek, is always implied.

Let $\gamma$ be a linear connexion on $M$. If $\Gamma_{j i}{ }^{h}(1 \leqq a, h, i, j, \cdots, \leqq n)$ are the components of $\gamma$ in the local coordinate system $\left(U, u^{h}\right)$ in $M$, then the components in $\left(U, u^{h}\right)$ of the torsion tensor $T$ and the curvature tensor $R$ on $M$ are respectively

$$
\begin{aligned}
T_{j i}{ }^{h} & =\Gamma_{j i}{ }^{h}-\Gamma_{i j}{ }^{h}, \\
R_{k j i}{ }^{h} & =\partial_{k} \Gamma_{j i}{ }^{h}-\partial_{j} \Gamma_{k i}{ }^{h}+\Gamma_{k a}{ }^{h} \Gamma_{j i}{ }^{a}-\Gamma_{j a}{ }^{h} \Gamma_{k i}{ }^{a},
\end{aligned}
$$

where $\partial_{k}=\partial / \partial u^{k}$. If $\gamma$ has zero torsion, i.e., if $T=0$, then the curvature tensor $R$ satisfies the two Bianchi identities

$$
\begin{aligned}
R_{k j i}{ }^{h}+R_{j i k}{ }^{h}+R_{i k j}{ }^{h} & =0 \\
\nabla_{l} R_{k j i}{ }^{h}+\nabla_{k} R_{j l i}{ }^{k}+\nabla_{j} R_{l k i}{ }^{h} & =0
\end{aligned}
$$

where $\nabla$ denotes covariant differentiation with respect to $\gamma$.

A linear connexion $\gamma$ on $M$ is said to be with recurrent curvature if its curvature tensor $R$ is not everywhere zero and if the covariant derivative of $R$ is equal to the tensor product of a covector and $R$ itself. We express these conditions in symbol by $R \neq \equiv$ and $\nabla R=W \otimes R$, and call $W$ the recurrence covector. By Theorem 1.2, the conditions $R \neq \equiv$ and $\nabla R=W \otimes R$ imply that $R \neq 0$. Hence for a linear connexion with recurrent curvature, the curvature tensor is nowhere zero, i.e. $R \neq 0$. In what follows, whenever the condition $\nabla R=W \otimes R$ is assumed, the condition $R \not \equiv 0$ and consequently also the condition $R \neq 0$ are always understood.

Recently, generalizing a result of K. Nomizu concerning linear connexions

Received by the editors May 18, 1961. 
with $\nabla R=0$, the author obtained a geometrical condition, in terms of the basic and fundamental vector fields on the frame bundle over $M$, for a linear connexion on $M$ to be with recurrent curvature (Wong [13, Theorem 4.2]). Except for this general result, linear connexions with recurrent curvature have so far been studied only in the following special cases:

1. Riemannian connexions $\left({ }^{1}\right)$ with $\nabla R=0$ (i.e. E. Cartan's symmetric spaces).

2. Linear connexions with $T=0, \nabla R=0$, or with $\nabla T=0, \nabla R=0$ (Nomizu $[3 ; 4])$.

3. Riemannian connexions $\left({ }^{1}\right)$ with $\nabla R=W \otimes R, W \neq 0$ (Ruse [5], Walker [8], Hlavaty [2]).

4. Certain type of linear connexions with $T=0, \nabla R=W \otimes R$ and $W \neq 0$ which include, except for a trivial case, all the Riemannian connexions in Case 3 above (Wong [9]).

5. Subflat linear connexions (i.e. linear connexions which admit locally $n-1$ parallel fields of vectors and $n-1$ parallel fields of covectors) with $T=0, \nabla R=W \otimes R$ and $W \neq 0$ (Wong [11]).

6. Projectively flat connexions with $T=0, \nabla R=W \otimes R$, and $W \neq 0$ or $W=0$ (Wong and Yano [12]).

Furthermore, in Cases 3-6, only local properties were considered.

In this paper, we shall study the more general case of linear connexions with $T=0, \nabla R=W \otimes R, W \not \equiv 0$, i.e. linear connexions with zero torsion and recurrent curvature for which the recurrence covector $W$ is not everywhere zero. Both local and global properties are obtained. The method we use is a combination of the classical tensor calculus and a method of piecing together locally defined tensor fields or fields of planes into global tensor fields or fields of planes on $M$. On two occasions, we apply Steenrod's theorem on existence of cross-sections in fiber bundles. It appears that neither the index-free presentation nor the method of exterior forms lends itself to the study of problems of the type considered in this paper. We assume that $n>2$. The case $n=2$ requires special treatment and will be considered in another paper (Wong [14]).

$\$ \S 1.1-1.3$ are preliminary in nature. They contain a number of results concerning parallel fields of planes or coplanes on $M$, recurrent tensors, and certain decomposition of recurrent tensors. All of them will play an important part in our later work. In $\$ \S 2.1-2.4$, we give a few rather easy but indispensable results on linear connexions with zero torsion and recurrent curvature without assuming that the recurrence covector $W$ is not everywhere zero.

Beginning with $\S 3$ is the study of linear connexions with zero torsion and

(1) Throughout this paper, the Riemannian connexions mentioned are those with a definite or indefinite metric. While Riemannian connexions with a positive definite metric and $\nabla R=0$ were described by E. Cartan as "une classe remarquable d'espaces de Riemann," the interesting case of Riemannian connexions with $\nabla R=W \otimes R$ and $W \not \equiv$ is when the metric is indefinite. 
recurrent curvature for which the recurrence covector $W$ is not everywhere zero. In $\$ 3.1$ we give some examples of such linear connexions showing the nature of the subset $M_{0}=\{u \in M: W(u)=0\}$. In $\S \S 3.2-3.3$ we prove among other things that the tensor $\nabla W$ is either everywhere symmetric or nowhere symmetric, and that the holonomy group can, and can only, be of dimension $1,2, \cdots, n-1$. In $\S \S 4.1-4.3$ we study the curvature tensor $R$ in detail. Local and global decompositions of $R$ in $M \backslash M_{0}$ (i.e. in the complement of $M_{0}$ in $M$ ) are obtained which give rise to a number of local fields of vectors and covectors in $M \backslash M_{0}$. Existence of certain global parallel fields of planes or coplanes spanned by these local vectors or covectors are established in $\S \S 5.1-5.3$. There we also prove that the Ricci tensor is of (constant) rank $\leqq 2$, and that in the case where the tensor $\nabla W$ is symmetric, the condition for the holonomy group to be abelian is expressible in terms of the pseudoorthogonality between certain parallel fields of planes and coplanes. The paper ends in $\S \S 6.1-6.3$ with a study of a decomposition of the tensor $\nabla W$ and the fields of covectors or coplanes arising from it.

In the course of our investigation, it is found that the properties of the linear connexion are quite different in the two cases where the dimension of the holonomy group is greater than 1 or equal to 1 . The latter case corresponds to the "simple" type (Walker [8]) of Riemannian connexion with recurrent curvature. It would be interesting to specialize the results presented in this paper to the case of Riemannian connexions and compare the results thus obtained with the earlier local results of H. S. Ruse, A. G. Walker and V. Hlavaty.

The author wishes to express his sincere thanks to his young colleague Mr. C. S. Hsü who read through a preliminary draft of this paper and suggested a number of improvements. Equation (3.1) of Theorem 3.1, part (a) of Theorem 4.2 and the global decomposition $\nabla W=N \otimes W+\tilde{N} \otimes \tilde{W}$ in Theorem 6.4 are partly due to him.

\section{Preliminaries}

Parallel fields of planes and recurrent tensors on a $C^{\infty}$-manifold with a linear connexion have been studied by A. G. Walker [7] and the author (Wong $[10 ; 13]$ ). Here, emphasizing the global aspects, we summarize a number of definitions and known results and add a few new ones for later use.

1.1. Parallel fields of vectors along a curve and parallel fields of planes or coplanes on $M$. Let $M$ be a connected, $n$-dimensional $C^{\infty}$-manifold (which consequently is also arcwise connected). A plane (resp. coplane) at a point $u \in M$ is a linear space spanned by a number of vectors (resp. covectors) at $u$. A field of planes (resp. coplanes) on $M$ is a $C^{\infty}$-assignment of a plane (resp. coplane) to each point of $M$.

Let $C: u=u(\tau), 0 \leqq \tau \leqq 1$, be a sectionally $C^{\infty}$-curve in $M$. It is easy to see that $C$ can be divided into a finite number of $C^{\infty}$-sections each of which lies 
in a coordinate neighborhood. Let $\left(U, u^{h}\right)$ be any one of these coordinate neighborhoods, and $u^{h}=u^{h}(\tau), \tau_{1} \leqq \tau \leqq \tau_{2}$, the $C^{\infty}$-section of $C$ lying in $U$. Then a vector field $X(\tau)$ defined on $C$ is said to be parallel along $C$ if the components $X^{h}(\tau)$ of $X(\tau)$ in each $\left(U, u^{h}\right)$ satisfy the linear differential equations

$$
\frac{d u^{l}}{d \tau} \nabla_{l} X^{h}=0 \text {, i.e. } \frac{d X^{h}}{d \tau}+\frac{d u^{j}}{d \tau} \Gamma_{j i}^{h} X^{i}=0 \quad\left(\tau_{1} \leqq \tau \leqq \tau_{2}\right) .
$$

The following lemmas are easy consequences of well-known properties of the solutions of systems of linear differential equations:

Lemma 1.1. For any given vector $A$ at the point $u(0)$, there exists a unique parallel vector field $X(\tau)$ along $C$ such that $X(0)=A$.

Lemma 1.2. If $X_{\alpha}(1 \leqq \alpha \leqq n)$ are any $n$ parallel vector fields along $C$ and $X_{\alpha}$ are independent at some point of $C$, then $X_{\alpha}$ are everywhere independent along $C$. In this case, any parallel vector field $X$ along $C$ can be expressed uniquely as $X=c^{\alpha} X_{\alpha}$, where $c^{\alpha}$ are constants.

Lemma 1.3. If $X_{A}(1 \leqq A \leqq r)$ are any $r$ parallel vector fields along $C$, then the number of independent vectors among $X_{A}(\tau)$ at any point of $C$ is constant along $C$.

A field $D$ of planes on $M$ is parallel if, for any two points $u_{0}, u_{1} \in M$, a vector in $D\left(u_{0}\right)$ is displaced parallelly along any (sectionally $C^{\infty}$ ) curve joining $u_{0}$ to $u_{1}$ into a vector in $D\left(u_{1}\right)$. Covector fields which are parallel along a curve and parallel fields of coplanes on $M$ are defined in a similar way.

On account of Lemma 1.3 , the planes of a parallel field $D$ on $M$ must be of the same dimension everywhere on $M$. Thus, if we wish to indicate the dimension of the planes, we can say that $D$ is a parallel field of $r$-planes.

Using Lemma 1.3, we can easily prove

Lemma 1.4. Let $D_{1}$ be a parallel field of $r_{1}$-planes and $D_{2}$ a parallel field of $r_{2}$-planes on $M$. Then the intersection planes $D_{1}(u) \cap D_{2}(u)$ at all points $u \in M$ are of the same dimension, and they form a parallel field of planes on $M$. The same is also true of the union planes $D_{1}(u) \cup D_{2}(u)$.

The following well-known result can be proved by modifying Walker's [7, Theorem 3.3] original proof in the case of Riemannian connexion:

Lemma 1.5. A field $D$ of $r$-planes on $M$ is parallel iff in each coordinate neighborhood $U \subset M$ and for any local basis $X_{A}^{h}(1 \leqq A, B \leqq r)$ of $D$ in $U$,

$$
\nabla_{l} X_{B}^{h}=L_{l B}^{A} X_{A}^{h},
$$

where $L_{l B}^{A}$ are some covectors on $U$.

We are now ready to prove our main result in this paragraph. 
TheOREM 1.1. Let $r$ be a fixed positive number. If for each point $u$ in $M$, there exists some coordinate neighborhood $U \ni u$ in $M$, and a set of $r$ vectors $Y_{\Lambda}^{h}(1 \leqq A \leqq r)$ on $U$ such that

$$
\text { on } U: \nabla_{l} Y_{A}^{h}=L_{l A}^{B} Y_{B}^{h} \quad(1 \leqq A, B \leqq r),
$$

and

$$
\text { on } U \cap U^{*}: Y_{A^{*}}^{* h}=\phi_{A^{*}}^{A} Y_{A}^{h}, \quad Y_{A}^{h}=\phi_{A}^{A^{*}} Y_{A^{*}}^{* h} \quad\left(1 \leqq A, A^{*} \leqq r\right),
$$

where $U^{*}$ is another coordinate neighborhood and $\phi_{A^{*}}^{A^{*}}, \phi_{A}^{A^{*}}$ are functions on $U \cap U^{*}$, then the local fields of planes spanned by these sets of local vector fields piece together into a parallel field of planes on $M$.

Proof. Condition (1.2) assures us that the local fields of planes spanned by the sets of local vector fields piece together to form a field of planes on $M$. Therefore, on account of Lemma 1.5, it is sufficient to prove that if the $r$ vectors $Y_{A}^{h}$ on $U$ satisfy condition (1.1), the number of independent vectors among them is the same everywhere in $U$.

To prove this, consider in $U$ any two points $u_{0}, u_{1}$, and some curve $u(\tau)$, $0 \leqq \tau \leqq 1$, joining $u_{0}=u(0)$ to $u_{1}=u(1)$. Let $X_{h}^{\alpha}(1 \leqq \alpha \leqq n)$ be some parallel coframe along $u(\tau)$, and define the functions $f_{A}^{\alpha}=Y_{A}^{h} X_{h}^{\alpha}$ on $u(\tau)$. We have, by condition (1.1),

$$
\begin{aligned}
d_{\tau}\left(Y_{A}^{h} X_{h}^{\alpha}\right) & =\delta_{\tau}\left(Y_{A}^{h} X_{h}^{\alpha}\right)=\left(\delta_{\tau} Y_{A}^{h}\right) X_{h}^{\alpha} \\
& =\left(d_{\tau} u^{l}\right) L_{l A}^{B} Y_{B}^{h} X_{h}^{\alpha},
\end{aligned}
$$

where $d_{\tau}=d / d \tau$ and $\delta_{\tau}=\left(d_{\tau} u^{l}\right) \nabla_{l}$. Therefore, the functions $f_{\boldsymbol{\Lambda}}^{\alpha}$ satisfy the equations

$$
d_{\tau} f_{A}^{\alpha}=\left(d_{\tau} u^{l}\right) L_{l A}^{B} f_{B}^{\alpha}
$$

In other words, $f_{A}^{\alpha}(\tau), 1 \leqq \alpha \leqq n$, are $n$ solutions of the system of linear differential equations

$$
d_{\tau} f_{A}=\left(d_{\tau} u^{l}\right) L_{l A}^{B} f_{B}
$$

in the $r$ unknown functions $f_{A}(1 \leqq A \leqq r)$.

Let $\bar{f}_{A}^{B}(1 \leqq B \leqq r)$ be any $r$ independent solutions of this system of linear differential equations. Then $f_{A}^{\alpha}=\bar{f}_{A}^{B} c_{B}^{\alpha}$, where $c_{B}^{\alpha}$ are constants. Therefore, since the matrix $\left(\bar{f}_{A}^{B}\right)$ is of rank $r$ all along $u(\tau)$ (from the theory of linear differential equations),

$$
\operatorname{rank}\left(f_{A}^{\alpha}\right)=\operatorname{rank}\left(c_{B}^{\alpha}\right)=\text { constant along } u(\tau) .
$$


But $Y_{\Lambda}^{h} X_{h}^{\alpha}=f_{\Lambda}^{\alpha}$ and matrix $\left(X_{h}^{\alpha}\right)$ is of rank $n$ all along $u(\tau)$. Therefore, rank $\left(Y_{A}^{h}\right)=\operatorname{rank}\left(f_{A}^{\alpha}\right)=$ constant along $u(\tau)$. Hence

$$
\operatorname{rank}\left(Y_{A}^{h}\right)_{u_{0}}=\operatorname{rank}\left(Y_{A}^{h}\right)_{u_{1}},
$$

as was to be proved.

It is easy to see that results similar to Lemmas 1.1-1.5 and Theorem 1.1 hold for covector fields and fields of coplanes.

A vector $X^{h}$ and a covector $Y_{h}$ at a point $u \in M$ are said to be pseudoorthogonal to each other if $X^{h} Y_{h}=0$. Pseudo-orthogonality between an $r$ plane and an $r^{\prime}$-coplane at a point is defined in an obvious manner. Moreover, it is easy to prove

LEMMA 1.6. Let $D$ be a parallel field of $r$-planes on $M$ and $D^{\prime}$ a parallel field of $r^{\prime}$-coplanes on $M$. Then if $D$ and $D^{\prime}$ are pseudo-orthogonal at some point of $M$, they are pseudo-orthogonal at every point of $M$.

Lemma 1.7. Let $D$ be a field of $r$-planes on $M$ and $D^{\prime}$ the field of $(n-r)$ coplanes pseudo-orthogonal to $D$. Then if $D$ is parallel, $D^{\prime}$ is also parallel, and conversely.

1.2. Recurrent tensors and holonomy group of a linear connexion with recurrent curvature. Let $M$ be a connected $n$-dimensional $C^{\infty}$-manifold on which a linear connexion has been given. Then we have

THEOREM 1.2. If $S$ is any tensor, say of type $(1,3)$, on $M$ satisfying the condition $\nabla S=W \otimes S$, then

(a) To each point $u$ of $M$, we can assign (not necessarily in a continuous manner) a frame $X_{\alpha}^{h}(u)$ such that the set of $n^{4}$ numbers

$$
S_{\beta \alpha \mu}^{\lambda}(u)=\left(S_{k j i}{ }^{h} X_{\beta}^{k} X_{\alpha}^{j} X_{\mu}^{i} X_{h}^{\lambda}\right)(u),
$$

which are functions of $u$, are proportional to a set of $n^{4}$ constants.

(b) $S$ is either everywhere zero or nowhere zero.

A tensor $S$ on $M$ is said to be recurrent if it satisfies the condition $\nabla S$ $=W \otimes S$ without being zero everywhere. Then by Theorem 1.2 (b),

CoRollaRy. A recurrent tensor is nowhere zero.

Proof of Theorem 1.2. The frame that we assign to each point of $M$ is constructed as follows. Fix any point $u_{0} \in M$ and assign to it any fixed frame $X_{\alpha}\left(u_{0}\right)$. For each point $u_{1}$ of $M$, fix a sectionally $C^{\infty}$-curve $C_{1}: u=u(\tau)$, $-\epsilon \leqq \tau \leqq 1+\epsilon$, passing through the points $u_{0}=u(0), u_{1}=u(1)$. By displacing the frame $X_{\alpha}\left(u_{0}\right)$ at $u_{0}$ parallelly along $C_{1}$, we obtain a field of frames $Y_{\alpha}(\tau)$ along $C_{1}$. Then $Y_{\alpha}(1)$ is the frame $X_{\alpha}\left(u_{1}\right)$ that we assign to the point $u_{1}$. To prove (a) we have to prove that the two sets of numbers 


$$
S_{\beta \alpha \mu}{ }^{\lambda}\left(u_{1}\right), \quad S_{\beta \alpha \mu}{ }^{\lambda}\left(u_{0}\right)
$$

constructed by using the frames $X_{\alpha}\left(u_{1}\right)$ and $X_{\alpha}\left(u_{0}\right)$ in accordance with (1.3) are proportional. For this purpose, we now prove that the set of numbers

$$
\tilde{S}_{\beta \alpha \mu}{ }^{\lambda}(\tau)=\left(S_{k j i}{ }^{h}(u) Y_{\beta}^{k} Y_{\alpha}^{j} Y_{\mu}^{i} Y_{h}^{\lambda}\right)(\tau)
$$

defined at each point of $C_{1}$ are always proportional to the set of numbers $\tilde{S}_{\beta \alpha \mu}{ }^{\lambda}(0)$. Since the sectionally $C^{\infty}$-curve $C_{1}$ can be divided into a finite number of $C^{\infty}$-sections each lying in a coordinate neighborhood, it suffices to prove the last assertion for the case when the points $u_{0}, u_{1}$ lie in the same coordinate neighborhood and the curve $C_{1}$ through them is $C^{\infty}$. Since $Y_{\alpha}(\tau)$ is parallel along $C_{1}$ and $S$ satisfies the condition $\nabla S=W \otimes S$, we have

$$
\begin{aligned}
d_{\tau} \tilde{S}_{\beta \alpha \mu}^{\lambda} & =\left(d_{\tau} u^{l}\right) \nabla_{W} \tilde{S}_{\beta \alpha \mu}^{\lambda} \\
& =\left(d_{\tau} u^{l}\right) \nabla_{l} S_{k j i}{ }^{h}(u) Y_{\beta}^{k} Y_{\alpha}^{j} Y_{\mu}^{i} Y_{h}^{\lambda} \\
& =\left(d_{\tau} u^{l}\right) W_{l}(u) S_{k j i}{ }^{h}(u) Y_{\beta}^{k} Y_{\alpha}^{j} Y_{\mu}^{i} Y_{h}^{\lambda}
\end{aligned}
$$

i.e.,

$$
d_{\tau} \tilde{S}_{\beta \alpha \mu}{ }^{\lambda}=\left(d_{\tau} u^{l}\right) W_{l}(u) \tilde{S}_{\beta \alpha \mu}{ }^{\lambda} .
$$

From this it follows that

$$
\tilde{S}_{\beta \alpha \mu}{ }^{\lambda}(\tau)=\tilde{S}_{\beta \alpha \mu}{ }^{\lambda}(0) \exp \int_{0}^{\tau}\left(d_{\tau} u^{l}\right) W_{l}(u) d \tau .
$$

This shows that the set of $n^{4}$ numbers $S_{\beta \alpha \mu}{ }^{\lambda}\left(u_{1}\right)=\tilde{S}_{\beta \alpha \mu}{ }^{\lambda}(1)$ are proportional to the set of $n^{4}$ numbers $S_{\beta \alpha \mu}{ }^{\lambda}\left(u_{0}\right)=\widetilde{S}_{\beta \alpha \mu}{ }^{\lambda}(0)$, which proves (a). In particular, if all the $n^{4}$ numbers $S_{\beta \alpha \mu}{ }^{\lambda}\left(u_{0}\right)$ are zero, so are also all the $n^{4}$ numbers $S_{\beta \alpha \mu}{ }^{\lambda}\left(u_{1}\right)$; in other words, if the tensor $S\left(u_{0}\right)$ is zero, so is the tensor $S\left(u_{1}\right)$. This proves (b).

Let $u$ be a point in $M$ and $C$ a loop at $u$ (i.e. a sectionally $C^{\infty}$ closed curve beginning and ending at $u)$. If we take any frame $\left(u, X_{\alpha}\right)$ at $u$ and transport it parallelly along $C$ once to arrive at the frame $\left(u, X_{\alpha}^{\prime}\right)$ at $u$, then the linear transformation $\sigma_{C}$ in the tangent $n$-plane to $M$ at $u$ which carries the frame $\left(u, X_{\alpha}\right)$ into the frame $\left(u, X_{\alpha}^{\prime}\right)$ is independent of the choice of the initial frame $\left(u, X_{\alpha}\right)$. For a fixed point $u$ in $M$, the set of linear transformations $\sigma_{C}$ corresponding to all the possible loops at $u$ form a subgroup of the general linear group $G L(n, R)$. This subgroup is the holonomy group of the linear connexion with reference point $u$. It follows at once from definition that the holonomy group at $u \in M$ leaves invariant an $r$-plane of the tangent $n$-plane to $M$ at $u$ iff there exists a parallel field of $r$-planes on $M$. Since the holonomy groups with different reference points are isomorphic, the reference point will not be mentioned specifically. 
A linear connexion on $M$ is said to be with recurrent curvature if its curvature tensor is recurrent. The following theorem is known (Wong [13, Theorem 4.3]):

THEOREM 1.3. For a linear connexion with recurrent curvature on a connected $n$-dimensional $C^{\infty}$-manifold $M$, the Lie algebra of its holonomy group $H$ is spanned by the following elements (which are $n \times n$ matrices) of the Lie algebra of $G L(n, R)$ :

$$
\operatorname{matrix}\left[R_{(\beta \alpha) \mu}{ }^{\lambda}(u)\right], \quad 1 \leqq \beta, \alpha \leqq n,
$$

where

$$
R_{(\beta \alpha) \mu}^{\lambda}(u)=\left(R_{k j i}{ }^{h} X_{\beta}^{k} X_{\alpha}^{j} X_{\mu}^{i} X_{h}^{\lambda}\right)(u),
$$

$u$ is any point in $M$ and $X_{\alpha}^{i}$ any frame at $u$. Consequently, the dimension of the holonomy group $H$ is equal to the number of independent ones among the matrices $\left[R_{(\beta \alpha) \mu}^{\lambda}(u)\right]$.

It follows from this that the holonomy group of a linear connexion with recurrent curvature on $M$ is at most of dimension $n(n-1) / 2$.

1.3. Local decomposition of a recurrent tensor. The main result in this paragraph is Theorem 1.4 concerning a local decomposition of a given recurrent tensor into the sum of tensor products of tensors. That theorem and Lemma 1.8 hold for a tensor of any type and for any two complementary sets of indices, but for simplicity and in anticipation of our applications, we state and prove them for a tensor $S_{k j i}{ }^{h}$ of type $(1,3)$ and for the complementary sets of indices $k, j$ and $i, h$.

We first consider the case of tensors and vectors at a single point $u \in U \subset M$. Let $S_{k j i}{ }^{h}$ be any tensor of type $(1,3)$. In the linear space of tensors of type $(0,2)$, the linear subspace spanned by the $n^{2}$ tensors

$$
S_{k j \mu}{ }^{\lambda}=S_{k j i}{ }^{h} X_{\mu}^{i} X_{h}^{\lambda} \quad(1 \leqq \mu, \lambda \leqq n),
$$

where $X_{\alpha}^{i}$ is any frame and $X_{h}^{\alpha}$ its dual coframe, is obviously independent of the choice of the frame $X_{\alpha}^{i}$. We call this linear subspace the $(k, j)$-support of $S$, and its dimension the $(k, j)$-dimension of $S$. In an analogous manner, the $(i, h)$-support, $(i, h)$-dimension or the $j$-support, etc. of $S$ can be defined.

The following lemma is known and quite easy to prove (see, for example, Hlavaty $[1, \S 1])$.

LEMMA 1.8. Let $S_{k j i}{ }^{h}$ be a tensor of type $(1,3)$ at the point $u \in U \subset M$. Then

(a) The $(k, j)$-dimension $=$ the $(i, h)$-dimension.

(b) The $(k, j)$-dimension of $S$ is equal to $r$ iff $S$ can be expressed as

$$
S_{k j i}{ }^{h}=P_{k j}^{A} Q_{A i}{ }^{h} \quad(1 \leqq A \leqq r),
$$


where $P_{k j}^{\Lambda}$ (also $Q_{A}{ }^{h}$ ) are $r$ linearly independent tensors.

(c) If the tensor $S$ can also be expressed as

$$
S_{k j i}{ }^{h}=P_{k j}^{* A^{*}} Q_{A^{*} i}^{* h}
$$

where $P_{k j}^{* A^{*}}\left(\right.$ also $\left.Q_{A_{i}^{*}}^{*}\right)$ are $r^{*}$ linearly independent tensors, then $r^{*}=r$ and

$$
P_{k j}^{* A^{*}}=\phi_{A}^{A^{*}} P_{k j}^{A}, \quad Q_{A^{*} i}^{* h}=\phi_{A^{*}}^{A} Q_{A i}^{h},
$$

where $\phi_{A^{*}}^{A^{*}}, \phi_{A^{*}}^{A}$ are scalars satisfying $\phi_{A}^{A^{*}} \phi_{A^{*}}^{B}=\delta_{A^{B}}^{B}$.

(d) If

$$
S_{\beta \alpha \mu}{ }^{\lambda}=S_{k j i}^{h} X_{\beta}^{k} X_{\alpha}^{j} X_{\mu}^{i} X_{h}^{\lambda},
$$

where $X_{\alpha}^{h}$ is any frame and $X_{h}^{\alpha}$ its dual coframe, then the $(k, j)$-dimension (=the $(i, h)$-dimension) of $S$ is equal to the rank of the matrix

$$
\left[S_{\beta \alpha \mu}{ }^{\lambda}\right] \text {, }
$$

where $(\mu, \lambda)$ denote the row and $(\beta, \alpha)$ denote the column.

Proof. We omit the details of the proof, but merely indicate how the tensors $P_{k j}^{A}$ and $Q_{A i}{ }^{h}$ can be constructed. Let $r$ be the $(k, j)$-dimension of $S_{k j i}{ }^{h}$, and let $P_{k j}^{A}(1 \leqq A \leqq r)$ be a set of $r$ independent tensors among the $n^{2}$ tensors $S_{k j \mu}{ }^{\lambda}=S_{k j i}{ }^{h} X_{\mu}^{i} X_{h}^{\lambda}(1 \leqq \mu, \lambda \leqq n)$. Then there exist scalars $\rho_{A \mu}{ }^{\lambda}$ such that

$$
S_{k j \mu}^{\lambda}={ }_{\rho_{A \mu} \lambda}^{\lambda} P_{k j}^{A}
$$

and

$$
S_{k j i}{ }^{h}=\left({ }_{\rho_{A \mu}}^{\lambda} P_{k j}^{A}\right) X_{i}^{\mu} X_{\lambda}^{h}=P_{k j}^{A}\left(\rho_{A \mu}^{\lambda} X_{i}^{\mu} X_{\lambda}^{h}\right) .
$$

Thus, writing $\rho_{A \lambda}^{\mu} X_{i}^{\mu} X_{\lambda}^{h}$ as $Q_{A i}{ }^{h}$, we have a decomposition of the form (1.4).

We now consider the case of a recurrent tensor on $M$.

THEOREM 1.4. Let $S$ be a recurrent tensor of type $(1,3)$ on $M: S \neq 0, \nabla S$ $=W \otimes S$, and let the components of $S$ in any coordinate system $\left(U, u^{h}\right)$ be $S_{k j i}{ }^{h}$. Then we have

(a) The $(k, j)$-dimension of $S$ is constant on $M$; in other words, the $(k, j)$ dimension $r(u)$ of $S(u)$ defined at every point $u$ of $M$ is the same.

(b) In a suitable coordinate neighborhood $U$ of each point $u$ in $M, S$ can be decomposed into the sum of tensor products

$$
S_{k j i}{ }^{h}=P_{k j}^{A} Q_{A i}{ }^{h} \quad(1 \leqq A, B \leqq r),
$$

where the $r$ tensors $P_{k j}^{A}$ (also the $r$ tensors $Q_{A i}{ }^{h}$ ) are everywhere independent in $U$.

(c) The tensors $P_{k \rightarrow}^{A}$ and $Q_{A i}{ }^{h}$ satisfy the relations: 


$$
\begin{aligned}
\nabla_{l} P_{k j}^{A}= & L_{L B}^{A} P_{k j}^{B}, \quad \nabla_{l} Q_{B i}{ }^{h}=\tilde{L}_{l B}^{A} Q_{A i}{ }^{h}, \\
& L_{l B}^{A}+\tilde{L}_{l B}^{A}=W_{l \delta_{B},},
\end{aligned}
$$

where $L_{i B}^{A}, \tilde{L}_{l B}^{A}$ are some covectors.

(d) If on another coordinate neighborhood $U^{*} \ni u, S$ is decomposed into

$$
S_{k j i}{ }^{h}=P_{k j}^{* A^{*}} Q_{A^{*} i}{ }^{k} \quad\left(1 \leqq A, B, A^{*} \leqq r\right),
$$

then on $U \cap U^{*}$,

$$
P_{k j}^{* A^{*}}=P_{k j}^{A} \phi_{A}^{A^{*}}, \quad Q_{A^{*} i}^{* h}={ }_{\phi_{A}^{*}}^{A} Q_{A i}{ }^{h},
$$

where $\phi_{A}^{A^{*}}, \phi_{A^{*}}^{A}$ are scalars on $U \cap U^{*}$ such that $\phi_{A}^{A^{*}} \phi_{A^{*}}^{B}=\delta_{A^{B}}^{B}$.

Proof. By Lemma 1.8(d), the $(k, j)$-dimension $r(u)$ of the tensor $S(u)$ is equal to the rank of the matrix

$$
\left[S_{\beta \alpha \mu}{ }^{\lambda}(u)\right] \quad \text { with row }(\mu \lambda) \text { and column }(\beta \alpha),
$$

where $S_{\beta \alpha \mu}{ }^{\lambda}(u)=\left(S_{k j i}{ }^{h} X_{\beta}^{k} X_{\alpha}^{j} X_{\mu}^{i} X_{h}^{\lambda}\right)(u)$ and $X_{\alpha}^{i}$ is any frame at $u$. Since $S$ is recurrent, we can, by Theorem 1.2(a) and its corollary, assign a frame to each point $u$ of $M$ so that the set of numbers $S_{\beta \alpha \mu}{ }^{\lambda}(u)$, not all zero, are proportional to a set of constants, say $c_{\beta \alpha \mu}{ }^{\lambda}$, which are independent of $u$. Therefore, $r(u)$ is equal to the rank of the matrix $\left[c_{\beta \alpha \mu}{ }^{\lambda}\right]$ and is consequently constant on $M$. This proves (a).

To prove (b), we take any $C^{\infty}$ field of frames $X_{\alpha}^{i}$ in any coordinate neighborhood $\widetilde{U}$ containing $u$ and use it to construct the tensors $P_{k, j}^{A}, Q_{A i}{ }^{h}$ as in the proof of Lemma 1.8. These tensors are defined on $\widetilde{U}$ and are obviously $C^{\infty}$. They will satisfy the independence condition in some suitable neighborhood $U \subset \tilde{U}$ of $u$.

Finally, to prove (c), we differentiate (1.5) covariantly and get

$$
\left(\nabla_{l} P_{k j}^{A}\right) Q_{A i}{ }^{h}+P_{k j}^{A}\left(\nabla_{l} Q_{A i}{ }^{h}\right)=\nabla_{l} S_{k j i}{ }^{h}=W_{l} S_{k j i}{ }^{h}=W_{l} P_{k j}^{A} Q_{A i}{ }^{h} .
$$

Since the $r$ tensors $P_{k j}^{A}$ (also the $r$ tensors $Q_{A i}{ }^{h}$ ) are independent, there exist some tensors $\bar{P}_{B}^{k j}$ and $\bar{Q}_{n}^{B i}$ such that $P_{k j}^{A} \bar{P}_{B}^{k j}=\delta_{B}^{A}$ and $Q_{A i}{ }^{h} \bar{Q}_{n}^{B i}=\delta_{A}^{B}$. Contractions of equation (1.7) by $\bar{Q}_{h}^{B i}$ and $\bar{P}_{B}^{k j}$ respectively show that $\nabla_{l} P_{k j}^{A}$ and $\nabla_{l} Q_{B i}{ }^{h}$ are of the form

$$
\nabla_{l} P_{k j}^{A}=L_{l B}^{A} P_{k j}^{B}, \quad \nabla_{l} Q_{B i}{ }^{h}=\tilde{L}_{l B}^{A} Q_{A i}{ }^{h} .
$$

Substituting these in (1.7), we arrive at

$$
L_{l B}^{A}+\tilde{L}_{l B}^{A}=W_{l} \delta_{B}^{A}
$$

which completes the proof of the theorem. 
Theorem 1.4 holds for a recurrent tensor of any type and for any two complementary sets of indices. Thus, let $s$ be the $h$-dimension of $S$ and $Q_{A}{ }^{h}$ any $s$ vectors which span the $h$-support of $S$. Then $S$ can be locally decomposed in $U$ as

$$
S_{k j i}{ }^{h}=P_{k j i}^{A} Q_{A}{ }^{h}
$$

and the $s$ vectors $Q_{A}{ }^{h}$ satisfy the relations

$$
\nabla_{l} Q_{B}{ }^{h}=\tilde{L}_{l B}^{A} Q_{A}{ }^{h}
$$

Furthermore, if

$$
S_{k j i}{ }^{h}=P_{k j i}^{* A^{*}} Q_{A^{*}}^{* h} \quad\left(1 \leqq A^{*}, B^{*} \leqq s\right)
$$

is a local decomposition of $S$ on $U^{*}$, then on $U \cap U^{*}$,

$$
Q_{A^{*}}{ }^{h}={ }_{A_{A}^{*}}^{A} Q_{A}{ }^{h}, \quad Q_{A}{ }^{h}=\phi_{A}^{A^{*}} Q_{A^{*}}{ }^{h}
$$

Hence the local vectors $Q_{A}{ }^{h}$ have the properties of the vectors $Y_{A}{ }^{h}$ in Theorem 1.1 , and we obtain

COROLLARY. If $S$ is a recurrent tensor on $M$, and $h$ any of its contravariant (resp. covariant) index, then the h-dimension of $S$ is constant on $M$ and the $h$-support forms a parallel field of planes (resp. coplanes) on $M$.

\section{LINEAR CONNEXIONS WITH $\nabla R=W \otimes R$}

In this section, we give a few easy but indispensable results concerning linear connexions with recurrent curvature. Here we do not assume that the recurrence covector $W$ is not everywhere zero.

2.1. The Ricci tensor. The Ricci tensor is defined by $R_{j i}=R_{h j i}{ }^{h}$. We have

THEOREM 2.1. For a linear connexion with recurrent curvature (not necessarily without torsion),

(a) The Ricci tensor is everywhere symmetric (resp. skew-symmetric) or nowhere symmetric (resp. skew-symmetric).

(b) The Ricci tensor is of the constant rank on $M$; in particular, it is everywhere zero or nowhere zero ( $c f$. Theorem 5.2).

Proof. From

$$
\nabla_{l} R_{k j i}{ }^{h}=W_{l} R_{k j i}{ }^{h}
$$

we deduce

$$
\begin{gathered}
\nabla_{l} R_{j i}=W_{l} R_{j i}, \quad \nabla_{l}\left(R_{j i}-R_{i j}\right)=W_{l}\left(R_{j i}-R_{i j}\right) \\
\nabla_{l}\left(R_{j i}+R_{i j}\right)=W_{l}\left(R_{j i}+R_{i j}\right) .
\end{gathered}
$$


On account of Theorem 1.2(b), the last two equations imply (a) of the theorem. Next, since the rank of $R_{j i}$ is equal to the $i$-dimension of $R_{j i}$, (b) follows from the corollary to Theorem 1.4.

In this connection, it is useful to note that for any linear connexion with zero torsion,

$$
R_{k j h}{ }^{h}=-\left(R_{k j}-R_{j k}\right) .
$$

This is obtained by contracting the indices $h$ and $i$ in the first Bianchi identity (0.1).

2.2. Supports of indices of the curvature tensor (cf. $\$ 1.3$ ).

THEOREM 2.2. For the curvature tensor $R_{k j i}{ }^{h}$ of any linear connexion with zero torsion,

$$
j \text {-support }=k \text {-support }, \quad i \text {-support } \subset k \text {-support. }
$$

If the linear connexion is with recurrent curvature, the support of each of the indices of the curvature tensor $R_{k j i}{ }^{h}$ forms a parallel field of planes or coplanes on $M$.

Proof. The first assertion is a consequence of the identities $R_{k j i}{ }^{h}=-R_{j k i}{ }^{h}$ and (0.1) which give

$$
R_{\beta j \mu^{\lambda}}{ }^{\lambda}=-R_{j \beta \mu^{\lambda}}{ }^{\lambda}, \quad R_{\beta \alpha i}{ }^{\lambda}=-R_{\alpha i \beta^{\lambda}}+R_{i \beta \alpha^{\lambda}}{ }^{\lambda},
$$

where for example, $R_{\beta j \mu}{ }^{\lambda}=R_{k j i}{ }^{h} X_{\beta}^{k} X_{\mu}^{i} X_{k}^{\lambda}$. The second assertion is a particular case of the Corollary to Theorem 1.4.

\subsection{The holonomy group.}

THEOREM 2.3. For a linear connexion with recurrent curvature (not necessarily with zero torsion), the $(k, j)$-dimension of its curvature tensor $R$ is constant on $M$. If the $(k, j)$-dimension of $R$ is $r$, and $R$ is decomposed at any point $u \in M$ into

$$
R_{k j i}{ }^{h}=P_{k j}^{A} Q_{A i}{ }^{h}
$$

$(1 \leqq A \leqq r)$,

then the Lie algebra of the holonomy group $H$ is spanned by the $r$ independent elements: matrix $\left[Q_{A_{\mu}{ }^{\lambda}}\right]$, where $Q_{A_{\mu}{ }^{\lambda}}=Q_{A_{i}}{ }^{h} X_{\mu}^{i} X_{h}^{\lambda}$ and $X_{\alpha}^{i}$ is any frame at $u$. Consequently, $\operatorname{dim} H=r$.

Proof. The first assertion is a particular case of Theorem 1.4. To prove the second assertion, we write

$$
R_{\beta \alpha \mu}{ }^{\lambda}=R_{k j i}{ }^{h} X_{\beta}^{k} X_{\alpha}^{j} X_{\mu}^{i} X_{h}^{\lambda}=\left(P_{k j}^{A} X_{\beta}^{k} X_{\alpha}^{j}\right)\left(Q_{A i}{ }^{h} X_{\mu}^{i} X_{h}^{\lambda}\right)
$$

as

$$
R_{\beta \alpha \mu}{ }^{\lambda}=P_{\beta \alpha}^{\Lambda} Q_{A \mu}{ }^{\lambda} .
$$


By Theorem 1.3, the Lie algebra of the holonomy group is spanned by the $n(n-1) / 2$ elements

$$
\operatorname{matrix}\left[R_{(\beta \alpha) \mu}{ }^{\lambda}\right]=P_{\beta \alpha}^{A} \operatorname{matrix}\left[Q_{A \mu}{ }^{\lambda}\right] \text {. }
$$

Since the $r$ tensors $Q_{A i}{ }^{h}$ (also the $r$ tensors $P_{k j}^{A}$ ) at $u$ are independent (cf. Theorem 1.4), it is easily seen from the above equation that the Lie algebra of $H$ is spanned by the $r$ independent elements: matrix $\left[Q_{A \mu}{ }^{\lambda}\right]$, as was to be proved.

2.4. A local decomposition of the curvature tensor.

THEOREM 2.4. For a linear connexion with zero torsion and recurrent curvature: $T=0, \nabla R=W \otimes R$, if $r$ is the dimension of the holonomy group, then in a suitable coordinate neighborhood $U$ of each point, the curvature tensor can be decomposed as

$$
R_{k j i}{ }^{h}=P_{k j}^{A} Q_{A i}{ }^{h} \quad(1 \leqq A, B, C, D \leqq r),
$$

where the $r$ tensors $P_{k j}^{A}$ (also the $r$ tensors $Q_{A i}{ }^{h}$ ) are everywhere independent in $U$ and satisfy the following relations:

$$
\begin{array}{rlrl}
\nabla_{l} P_{k j}^{A}= & L_{l B}^{A} P_{k j}^{B}, & \nabla_{l} Q_{B i}{ }^{h}=\tilde{L}_{l B}^{A} Q_{A i}{ }^{h}, & L_{l B}^{A}+\tilde{L}_{l B}^{A}=W_{l} \delta_{B}^{A} ; \\
P_{a[k}^{A} Q_{B j]}{ }^{a} & =C_{B C}^{A} P_{k j}^{C}, & \nabla_{[m} L_{l] B}^{A}+L_{[m B}^{D} L_{l] D}^{A}=C_{C B}^{A} P_{m l}^{C}, \\
Q_{[B a}{ }^{h} Q_{C] i}{ }^{a} & =\tilde{C}_{B C}^{A} Q_{A i}{ }^{h}, & \nabla_{[m} \tilde{L}_{l] B}^{A}+\tilde{L}_{[m D}^{A} \tilde{L}_{l] B}^{D}=\tilde{C}_{C B}^{A} P_{m l}^{C} ; \\
\left(\nabla_{[m} W_{l]}\right) \delta_{C}^{A}=\left(C_{B C}^{A}+\tilde{C}_{B C}^{A}\right) P_{m l}^{C}, & \nabla_{l} \tilde{C}_{B C}^{A}=\tilde{L}_{l[B}^{D} \tilde{C}_{D C]}^{A}-\tilde{L}_{l D}^{A} \tilde{C}_{B C}^{D} .
\end{array}
$$

Here $W_{l}$ is the recurrence covector, $L_{l B}^{A}$ and $\tilde{L}_{l B}^{A}$ are covectors, $C_{B C}^{A}$ and $\tilde{C}_{B C}^{A}\left(=-\tilde{C}_{C B}^{A}\right)$ are scalars, and square brackets used with indices indicate that alternation is taken over the two outermost indices; thus

$$
P_{a[k}^{A} Q_{B j]}{ }^{a} \equiv P_{a k}^{A} Q_{B j}{ }^{a}-P_{a j}^{A}-Q_{B k}{ }^{a} .
$$

Proof. The relations (2.3) have been proved in Theorem 1.4. We deduce from $(2.3)_{1}$ that

$$
\nabla_{[m} \nabla_{l]} P_{k j}^{A}=\left(\nabla_{[m} L_{l] B}^{A}+L_{[l C}^{A} L_{m] B}^{C}\right) P_{k j}^{B} .
$$

Applying to the left side the Ricci identity for a linear connexion with zero torsion, we have on account of (2.2)

$$
\begin{aligned}
\nabla_{[m} \nabla_{l]} P_{k j}^{A} & =-R_{m l k}{ }^{a} P_{a j}^{A}-R_{m l j}{ }^{a} P_{k a}^{A} \\
& =P_{m l}^{B}\left(-Q_{B k}{ }^{a} P_{a j}^{A}+Q_{B j}{ }^{a} P_{a k}^{A}\right) .
\end{aligned}
$$

Since $P_{k j}^{A}$ are independent tensors, comparison of the above two expressions for $\nabla_{[m} \nabla_{l]} P_{k j}^{A}$ gives $(2.4)_{1,2}$. Similarly, equations $(2.4)_{3,4}$ can be proved by 
using (2.2) and (2.3) $)_{2}$. Equation $(2.4)_{5}$ is an immediate consequence of $(2.3)_{3}$ and $(2.4)_{2,4}$. The last equation $(2.4)_{6}$ follows from $(2.3)_{2}$ and $(2.4)_{3}$.

We now prove

CoROllaRy. The holonomy group is abelian iff the functions $\tilde{C}_{B C}^{A}$ appearing in equation $(2.4)_{3}$ are all zero at some point of $M$ (then they are all zero everyi.here in $M$ ).

Proof. Let $X_{\alpha}^{i}$ be any frame at $u$ and let $Q_{\Delta_{\mu}{ }^{\lambda}}=Q_{A_{i}}{ }^{h}(u) X_{\mu}^{i} X_{h}^{\lambda}$. Then equation $(2.4)_{3}$ at $u$ is equivalent to

$$
Q_{[B \nu}{ }^{\lambda} Q_{C] \mu}{ }^{\nu}=\tilde{C}_{B C}^{A}(u) Q_{A \mu}{ }^{\lambda} .
$$

In consequence of Theorem 2.3, these are the structure equations of the holonomy group $H$ (with reference point $u$ ) so that $\widetilde{C}_{B C}^{A}(u)$ are the structural constants. Thus $H$ is abelian iff $\widetilde{C}_{B C}^{A}(u)=0$. Equation $(2.4)_{6}$ confirms the fact that if $\tilde{C}_{B C}^{A}$ are all zero at some point $u$ of $M$, they are all zero everywhere in $M$.

\section{Linear CONNEXIONS WiTh $T=0, \nabla R=W \otimes R, W \not \equiv 0$}

From now on we shall study exclusively linear connexions with zero torsion and recurrent curvature for which the recurrence covector $W$ is not everywhere zero. As before, the $n$-dimensional $C^{\infty}$-manifold $M$ on which such a linear connexion $\gamma$ is defined is assumed to be connected, and so arcwise connected.

Let $M_{0}=\{u \in M: W(u)=0\}$. If $M_{0}$ is an $n$-dimensional subset of $M$, as is the case in Example 1 in $\S 3.1$, then the restriction of $\gamma$ to $M_{0}$ is a linear connexion $\gamma_{0}$ with $T=0$ and $\nabla R=0$. Such a linear connexion $\gamma_{0}$ generalizes the symmetric (Riemannian) space of E. Cartan and has been studied quite extensively. But the subset $M_{0}$ may also be of dimension $n-1$ or $n-2$, as is the case in Examples 2 and 3 of $\S 3.1$. Apart from this, we know nothing about the nature of the subset $M_{0} \subset M$.

Let $M_{0}$ be as defined above, and let $M_{1}$ be any arcwise connected component of $M \backslash M_{0}$. Then $M_{1}$ is an $n$-dimensional connected $C^{\infty}$-manifold and the restriction of $\gamma$ to $M_{1}$ is a linear connexion $\gamma_{1}$ with $T=0, \nabla R=W \otimes R$ for which the recurrence covector $W$ is nowhere zero. Since the results in the previous sections were derived for a connected $C^{\infty}$-manifold we can apply them directly to $M$ and to each of the connected components of $M \backslash M_{0}$ but not to $M \backslash M_{0}$ which may not be connected. In what follows, we shall obtain various properties of the linear connexion $\gamma$ and properties of $M$ implied by the existence of $\gamma$ on it. Some of the results hold on the whole of $M$; others only on $M \backslash M_{0}$ or on any of its connected components; still a few others only on $\left(M \backslash M_{0}\right) \backslash M_{00}$, where $M_{00}=\left\{u \in\left(M \backslash M_{0}\right):\left[\left(\nabla_{l} W_{[k}\right) W_{j]}\right](u)=0\right\}$.

3.1. Some examples.

Example 1. On the Euclidean $n$-space $M:\left(-\infty<u^{1}, \cdots, u^{n}<+\infty\right)$, let 
$\gamma[\theta]$ denote the linear connexion (with zero torsion) defined by the components

$$
\begin{aligned}
\Gamma_{11}^{\xi} & =b_{\xi}^{\xi} u^{\zeta} \theta, \quad \Gamma_{\eta 1}{ }^{2}=\Gamma_{1 \eta}{ }^{2}=a_{\eta \xi} u^{\zeta} \theta, \\
\Gamma_{11}{ }^{2} & =\frac{1}{2} a_{\eta \zeta} u^{\eta} u^{\zeta} \frac{d \theta}{d u^{1}}+c_{\zeta} u^{\zeta} \theta, \quad(3 \leqq \xi, \eta, \zeta \leqq n),
\end{aligned}
$$

all the other $\Gamma_{j i}{ }^{h}$ are zero,

where the function $\theta=\theta\left(u^{1}\right)$ is nowhere zero, and $a_{7}, b^{\xi}, c_{5}$ are constants not all zero such that $a_{\eta \zeta}=a_{\zeta \eta}$ and $a_{\xi \eta} b_{\zeta}^{\xi}=a_{\xi \xi} b_{\eta}^{\xi}$.

By straightforward computation or from Wong [9], it can easily be verified that $\gamma[\theta]$ has recurrent curvature and the recurrence covector is $W_{l}$ $=\partial(\log \theta) / \partial u^{\imath}$.

Taking

$$
\theta= \begin{cases}1 & \text { for } u^{1} \leqq 0 \\ 1+\exp \left(-1 / u^{1}\right) & \text { for } u^{1}>0\end{cases}
$$

we obtain on $M$ a linear connexion with zero torsion and recurrent curvature for which $M_{0}=\{u \in M: W(u)=0\}$ is the subset $u^{1} \leqq 0$, and $M \backslash M_{0}$ is the connected subset $u^{1}>0$.

Example 2. Let $M$ and $\gamma[\theta]$ be as in Example 1. Taking $\theta=1+\left(u^{1}\right)^{2}$, we obtain on $M$ a linear connexion with zero torsion and recurrent curvature for which the subset $M_{0}$ is the $(n-1)$-plane $u^{1}=0$ and $M \backslash M_{0}$ consists of the two connected components $u^{1}<0$ and $u^{1}>0$.

Example 3. On the Euclidean $n$-space $M:\left(-\infty<u^{1}, \cdots, u^{n}<+\infty\right)$, let $\gamma[\phi]$ denote the linear connexion (with zero torsion) defined by the components

$$
\begin{aligned}
& \Gamma_{11}^{\xi}=b^{\xi} \partial \phi / \partial z, \quad \Gamma_{\eta 1}^{2}=\Gamma_{1 \eta}^{2}=a e_{\eta} \partial \phi / \partial z, \\
& \Gamma_{11}^{2}=a \partial \phi / \partial u^{1}+c \partial \phi / \partial z, \\
& \text { all the other } \Gamma_{j i}{ }^{h} \text { are zero, }
\end{aligned}
$$

where $a, b^{\xi}, c$ are constants not all zero; $e_{\zeta}$ are constants not all zero; $z=e_{\zeta} u^{\zeta}$, $\phi=\phi\left(u^{1}, z\right)$ is such that $\partial^{2} \phi / \partial z^{2}$ is nowhere zero. By straightforward computation or from Wong [9], it can easily be verified that $\gamma[\phi]$ has recurrent curvature and the recurrence covector is $W_{l}=\partial\left(\log \partial^{2} \phi / \partial z^{2}\right) / \partial u^{l}$. If we take as $\phi\left(u^{1}, z\right)$ any solution of the differential equation

$$
\partial^{2} \phi / \partial z^{2}=1+\left(u^{1}\right)^{2}+(z)^{2},
$$

we obtain on $M$ a linear connexion with zero torsion and recurrent curvature for which the subset $M_{0}$ is the $(n-2)$-plane: $u^{1}=0=z$, and $M \backslash M_{0}$ is a connected subset.

3.2. The recurrence covector $W$. We now prove 
THEOREM 3.1. For a linear connexion with $T=0, \nabla R=W \otimes R, W \neq 0$, the recurrence covector $W$ satisfies the equations

$$
\begin{gathered}
\nabla_{q}\left(\nabla_{[m} W_{l]}\right)=W_{q}\left(\nabla_{[m} W_{l]}\right), \\
W_{q} \nabla_{[m} W_{l]}+W_{m} \nabla_{[l} W_{q]}+W_{l} \nabla_{[q} W_{m]}=0 .
\end{gathered}
$$

Equation (3.1) implies that the tensor $\nabla W$ on $M$ is either everywhere symmetric or nowhere symmetric; in the former case, $W$ is locally a gradient. Equation (3.2) means that $W$ is locally proportional to a gradient; in other words, the total differential equation $W_{k} d u^{k}=0$ which is globally defined on $M$ is locally completely integrable.

Proof. On account of $\nabla R=W \otimes R$, we have

$$
\nabla_{m} \nabla_{l} R_{k j i}{ }^{h}=\nabla_{m}\left(W_{l} R_{k j i}{ }^{h}\right)=\left(\nabla_{m} W_{l}\right) R_{k j i}{ }^{h}+W_{m} W_{l} R_{k j i}{ }^{h} .
$$

Therefore, by the Ricci identity,

$$
\begin{aligned}
\left(\nabla_{[m} W_{l]}\right) R_{k j i}{ }^{h} & =\nabla_{[m} \nabla_{l]} R_{k j i}{ }^{h} \\
& =R_{m l p}{ }^{h} R_{k j i}{ }^{p}-R_{m l i}{ }^{p} R_{k j p}{ }^{h}-R_{m l j}{ }^{p} R_{k p i}{ }^{h}-R_{m l k}{ }^{p} R_{p j i}{ }^{h} .
\end{aligned}
$$

(It is easy to verify that this equation is equivalent to (2.4) $)_{\text {b.) }}$ Applying $\nabla_{q}$ to both sides of this equation and using the latter again in the result, we get

$$
\nabla_{q}\left(\nabla_{[m} W_{l]}\right) R_{k j i}{ }^{h}+W_{q}\left(\nabla_{[m} W_{l]}\right) R_{k j i}{ }^{h}=2 W_{q}\left(\nabla_{[m} W_{l]}\right) R_{k j i}{ }^{h},
$$

i.e.,

$$
\nabla_{Q}\left(\nabla_{[m} W_{l]}\right) R_{k j i}{ }^{h}=W_{Q}\left(\nabla_{[m} W_{l]}\right) R_{k j i}{ }^{h} .
$$

Since the curvature tensor is nowhere zero, it follows that

$$
\nabla_{q}\left(\nabla_{[m} W_{l]}\right)=W_{q}\left(\nabla_{[m} W_{l]}\right) \text {, }
$$

which proves (3.1). By Theorem 1.2(b), equation (3.1) implies that $\nabla_{[m} W_{l]}$ is either everywhere zero or nowhere zero.

To prove (3.2), let us denote by cyc( ) the cyclic expression with typical term inside the brackets. Then on account of (3.1), we have

$$
\begin{aligned}
\operatorname{cyc}\left(W_{q} \nabla_{[m} W_{l]}\right) & =\operatorname{cyc}\left(\nabla_{q} \nabla_{[m} W_{l]}\right)=\operatorname{cyc}\left(\nabla_{[m} \nabla_{l]} W_{q}\right) \\
& =\operatorname{cyc}\left(R_{m l q}{ }^{h} W_{h}\right) \\
& =\left(R_{m l q}{ }^{h}+R_{l q m}{ }^{h}+R_{q m l^{h}}\right) W_{h} \\
& =0 .
\end{aligned}
$$

This is (3.2) which is the well-known condition for $W$ to be locally proportional to a gradient. Hence our theorem is completely proved.

In consequence of Theorems 2.1 and 3.1, the Ricci tensor and the tensor $\nabla W$ share the property that it is either everywhere symmetric or nowhere symmetric. We observe also that 
(a) For a Riemannian connexion with $\nabla R=W \otimes R$ and $W \neq 0, \nabla W$ is symmetric (Walker $[8, \S 6]$ ).

(b) For a subflat or projectively flat connexion with $T=0, \nabla R=W \otimes R$ and $W \neq 0, \nabla W$ is symmetric iff the Ricci tensor is symmetric (Wong [11] and Wong and Yano [12]).

(c) For a 2-dimensional linear connexion with $T=0, \nabla R=W \otimes R$ and $W \not \equiv 0, \nabla W$ is symmetric iff the Ricci tensor is symmetric (Wong [14]). These facts support the truth of the following conjecture though the author has not been able to prove it.

CONJECTURE. For every linear connexion with $T=0, \nabla R=W \otimes R$ and $W \not \equiv 0$, the tensor $\nabla W$ is (everywhere) symmetric iff the Ricci tensor is (everywhere) symmetric.

\subsection{Dimension of the holonomy group.}

LEMMA 3.1. In any coordinate neighborhood $U$ in which $W$ has no zero, the curvature tensor can be expressed as

$$
R_{k j i}{ }^{h}=W_{k} S_{j i}{ }^{h}-W_{j} S_{k i}{ }^{h},
$$

where $S_{j i}{ }^{h}$ is some tensor on $U$.

Proof. Using $\nabla R=W \otimes R$ in the second Bianchi identity (0.2), we get

$$
W_{l} R_{k j i}{ }^{h}+W_{k} R_{j l i}{ }^{h}+W_{j} R_{l k i}{ }^{h}=0 .
$$

Since $W$ has no zero in $U$, there exists some vector $X^{l}$ on $U$ such that $X^{l} W_{l}$ $=1$. Contraction of the above equation by $X^{l}$ gives

$$
R_{k j i}{ }^{h}=W_{k} R_{l j i}{ }^{h} X^{l}-W_{j} R_{l k i}{ }^{h} X^{l},
$$

i.e.,

$$
R_{k j i}{ }^{h}=W_{k} S_{j i}{ }^{h}-W_{j} S_{k i}{ }^{h}
$$

where we have put

$$
S_{j i}{ }^{h}=R_{l j i}{ }^{h} X^{l} \text {. }
$$

We now prove

THEOREM 3.2. Let $H$ denote the holonomy group of a linear connexion with $T=0, \nabla R=W \cdot \otimes R$ and $W \not \equiv 0$. Then $1 \leqq \operatorname{dim} H \leqq n-1$. Moreover, for each integer $r$ satisfying $1 \leqq r \leqq n-1$, there exist linear connexions of this type for which $\operatorname{dim} H=r$.

Proof. Let us consider a point $u \in M$ at which $W \neq 0$. By Lemma 3.1, the curvature tensor is of the form

$$
R_{k j i}{ }^{h}=W_{k} S_{j i}{ }^{h}-W_{j} S_{k i}{ }^{h}
$$

at $u$. Choose a frame $X_{\alpha}^{h}$ at $u$ such that $W_{\beta}=W_{k} X_{\beta}^{k}=\delta_{\beta}^{1}$. Then the numbers $R_{(\beta \alpha)_{k}{ }^{\lambda}}(u)$ which appear in Theorem 1.3 are 


$$
R_{(\beta \alpha) \mu}{ }^{\lambda}(u)=\delta_{\beta \beta}^{1} S_{\alpha \mu}^{\lambda}(u)-\delta_{\alpha}{ }^{1} S_{\beta \mu}{ }^{\lambda}(u) .
$$

Consequently, among the $n(n-1) / 2$ matrices

$$
\left[R_{(\beta \alpha) \mu}{ }^{\lambda}(u)\right]
$$

only the following $n-1$ can possibly be independent:

$$
\left[R_{(1 \alpha) \mu}{ }^{\lambda}(u)\right]=\left[S_{(\alpha) \mu}{ }^{\lambda}(u)\right]
$$

Therefore, by Theorem 1.3, $\operatorname{dim} H \leqq n-1$. On the other hand, since the numbers $R_{(\beta \alpha) \mu^{\lambda}}(u)$ cannot all be zero, at least one of the above $n-1$ matrices is nonzero. Therefore $\operatorname{dim} H \geqq 1$.

To prove the second half of the theorem, we consider some examples. On the Euclidean $n$-space $M:\left(-\infty<u^{1}, \cdots, u^{n}<+\infty\right)$, let $\gamma$ be the linear connexion with zero torsion and recurrent curvature defined by the components

$$
\Gamma_{11}^{3}=\theta u^{3}, \cdots, \quad \Gamma_{11}^{r+2}=\theta u^{r+2}
$$

all the other $\Gamma_{j i}{ }^{h}$ are zero,

where $\theta=1+\left(u^{1}\right)^{2}$, and $r$ is any fixed integer such that $1 \leqq r \leqq n-2$. This is a special case of Example 2 in \$3.1. An easy computation will show that the only nonzero components of the curvature tensor are

$$
R_{1 j 1}{ }^{h}=\theta \delta_{j}^{h} \quad(3 \leqq h, j \leqq r+2)
$$

and those differing from these by sign. Taking $X_{\alpha}^{h}=\delta_{\alpha}^{h}$, we see by Theorem 1.3 that $\operatorname{dim} H$ is equal to the number of independent matrices among the following $r n \times n$ matrices:

$$
\left[R_{(1 \alpha) \mu}{ }^{\lambda}\right]=\theta\left[\delta_{\mu}^{1} \delta_{\alpha}^{\lambda}\right] \quad(\alpha=3, \cdots, r+2) .
$$

Now in the $\alpha$ th matrix the $(\alpha, 1)$-element is its only nonzero element. Therefore, these $r$ matrices are independent, and so $\operatorname{dim} H=r$. Hence there exist linear connexions of the type under considerations for which $\operatorname{dim} H=$ $1,2, \cdots, n-2$.

To give an example for which $\operatorname{dim} H=n-1$, let us consider on a suitable neighborhood $U$ in $M:\left(-\infty<u^{1}, \cdots, u^{n}<+\infty\right)$ a projectively flat connexion with recurrent curvature. In Wong [12], we have shown that for such a linear connexion, the curvature tensor is of the form (in suitable coordinates)

$$
R_{k j i}{ }^{h}=\theta\left(\delta_{k}^{h} \xi_{j}-\delta_{j}^{h} \xi_{k}\right) \xi_{i}
$$

or 


$$
R_{k j i}{ }^{h}=\psi\left[\left(\delta_{k}^{k} \xi_{j}-\delta_{j}{ }^{h} \xi_{k}\right) \eta_{i}-\left(\xi_{k} \eta_{j}-\xi_{j} \eta_{k}\right) \delta_{i}^{h}\right] \quad\left(\xi_{i}, \eta_{i}\right. \text { not proportional), }
$$

according as its Ricci tensor is symmetric or nonsymmetric.

For the case (3.3), let us choose a frame $X_{\alpha}^{h}$ at $u \in U$ such that $\xi_{\alpha}=\xi_{h} X_{\alpha}^{h}$ $=\delta_{\alpha}^{1}$. Then by Theorem 1.3, $\operatorname{dim} H$ is equal to the number of independent matrices among the following $n-1 n \times n$ matrices

$$
\left[R_{(\beta 1) \mu}{ }^{\lambda}(u)\right]=\theta\left[\delta_{\beta}^{\lambda} \xi_{\mu}(u)\right] \quad(\beta=2, \cdots, n) .
$$

But these $n-1$ matrices are all linearly independent since

$$
c^{\beta}\left(\delta_{\beta}^{\lambda} \xi_{\mu}\right)=0 \Rightarrow c^{\beta} \xi_{\mu}=0 \Rightarrow c^{\beta}=0 .
$$

Therefore, $\operatorname{dim} H=n-1$.

For the case (3.4), $\xi_{i}$ and $\eta_{i}$ are not proportional. Then using a frame $X_{\alpha}^{h}$ at $u \in U$ such that

$$
\xi_{\alpha}=\xi_{h} X_{\alpha}^{h}=\delta_{\alpha}^{1}, \quad \eta_{\alpha}=\eta_{h} X_{\alpha}^{h}=\delta_{\alpha}^{2},
$$

we can verify that in this case also $\operatorname{dim} H$ is equal to $n-1$.

Hence we have not only completed the proof of Theorem 3.5 but also proved the following

THEOREM 3.3. The holonomy group of an n-dimensional projectively flat connexion with $T=0, \nabla R=W \otimes R$ and $W \not \equiv 0$ is of dimension $n-1$.

In this connection, it is interesting to note that combining our Theorem 1.3 with a local result of Hlavaty [2, Theorem $3.4 \mathrm{a}, \mathrm{b}$ ] or with certain equivalent results of Walker ([8, (7.2) and p. 55]), we have

THEOREM 3.4. The holonomy group of an n-dimensional $(n>2)$ Riemannian connexion with $\nabla R=W \otimes R$ with $W \not \equiv 0$ is of dimension $\leqq n-2$.

\section{The CURVATURE TENSOR}

In this section, certain local and global decompositions of the curvature tensor $R$ into sums of tensor products are proved and studied in detail. In the course of our investigation, the distinction between the cases where the holonomy group is of dimension $>1$ or $=1$ arises naturally. In addition to being of interest on their own, the results obtained will form a basis of our work in the next two sections.

4.1. A local decomposition of $R$ in $M \backslash M_{0}$.

THEOREM 4.1. Let a linear connexion on $M$ be such that $T=0, \nabla R=W \otimes R$ and $W \not 0$ and let $M_{0}=\{u \in M: W(u)=0\}$. Then on each coordinate neighborhood $U$ in $M \backslash M_{0}$ there exists some tensor $S_{j i}{ }^{h}$, symmetric in $j, i$ and having no zero in $U$ such that $R$ is decomposed into 


$$
R_{k j i}{ }^{h}=W_{k} S_{j i}{ }^{h}-W_{j} S_{k i}{ }^{h} .
$$

The most general tensor $\widetilde{S}_{j i^{h}}$ having the properties of the tensor $S_{j i}{ }^{h}$ is of the form

$$
\widetilde{S}_{j i}{ }^{h}=S_{j i}{ }^{h}+W_{j} W_{i} C^{h},
$$

where $C^{h}$ is an arbitrary vector on $U$.

Proof. Since $W$ is nowhere zero in $M \backslash M_{0}$ it follows from Lemma 3.1 that on each coordinate neighborhood $U$ of $M \backslash M_{0}$ there exists some tensor $S$ of type $(1,2)$ such that

$$
R_{k j i}{ }^{h}=W_{k} S_{j i}{ }^{h}-W_{j} S_{k i}{ }^{h}
$$

holds.

If $\widetilde{S}$ is any tensor of type $(1,2)$ on $U$ satisfying

$$
R_{k j i}{ }^{h}=W_{k} \widetilde{S}_{j i}{ }^{h}-W_{j} \widetilde{S}_{k i}{ }^{h},
$$

then we derive from this and (4.1) that

$$
W_{k}\left(\tilde{S}_{j i}{ }^{h}-S_{j i}{ }^{h}\right)=W_{j}\left(\widetilde{S}_{k i}{ }^{h}-S_{k i}{ }^{h}\right),
$$

which gives (since $W$ has no zero in $U$ )

$$
\tilde{S}_{j i}{ }^{h}=S_{j i}{ }^{h}+W_{j} B_{i}{ }^{h}
$$

where $B_{i}{ }^{h}$ is some tensor of type $(1,1)$ on $U$.

Conversely, if $S_{j i}{ }^{h}$ is any tensor satisfying (4.1) and $B_{i}^{h}$ is any tensor of type $(1,1)$, then the tensor $\tilde{S}_{j i}{ }^{h}$ defined by (4.3) satisfies (4.1).

Now using (4.1) in the first Bianchi identity:

$$
R_{k j i}{ }^{h}+R_{j i k}^{h}+R_{i k j}^{h}=0,
$$

we obtain

$$
\left(W_{k} S_{j i}{ }^{h}-W_{j} S_{k i}{ }^{h}\right)+\left(W_{j} S_{i k}{ }^{h}-W_{i j} S_{j k}{ }^{h}\right)+\left(W_{i} S_{k j}{ }^{h}-W_{k} S_{i j}{ }^{h}\right)=0,
$$

i.e.

$$
W_{k} S_{[j i]^{h}}+W_{j} S_{[i k]^{h}}+W_{i} S_{[k j]^{h}}^{h}=0 .
$$

From this it follows easily that

$$
S_{j i}{ }^{h}-S_{i j}{ }^{h}=-W_{j} B_{i}{ }^{h}+W_{i} B_{j}{ }^{h}
$$

i.e.

$$
S_{j i}{ }^{h}+W_{j} B_{i}{ }^{h}=S_{i j}{ }^{h}+W_{i} B_{j}{ }^{h},
$$

where $B_{i}{ }^{h}$ is some tensor of type $(1,1)$ on $U$. Let us put $\tilde{S}_{j i}{ }^{h}=S_{j i}{ }^{h}+W_{j} B_{i}{ }^{h}$. Then on account of (4.4), the tensor $\tilde{S}_{j i}{ }^{h}$ is symmetric in $j, i$. Moreover, by

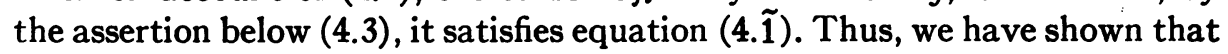
there exists a tensor having the properties of the tensor $S_{j i}{ }^{h}$ in the theorem. 
To complete the proof of the theorem, we see from (4.3) that the most general of such tensors is of the form

$$
\tilde{S}_{j i}{ }^{h}=S_{j i}{ }^{h}+W_{j} B_{i}{ }^{h} .
$$

But, because $\tilde{S}_{j i}{ }^{h}-S_{j i}{ }^{h}$ is symmetric in $j, i$, the tensor $B_{i}{ }^{h}$ must satisfy the equation $W_{j} B_{i}{ }^{h}=W_{i} B_{j}{ }^{h}$, and is therefore of the form $B_{i}{ }^{h}=W_{i} C^{h}$, where $C^{h}$ is an arbitrary vector. Hence, the most general tensor $\tilde{S}_{j i}{ }^{h}$ having the properties of the tensor $S_{j i}{ }^{h}$ in the theorem is

$$
\widetilde{S}_{j i}{ }^{h}=S_{j i}^{h}+W_{j} W_{i} C^{h},
$$

as was to be proved.

4.2. A global decomposition of the curvature tensor on $M \backslash M_{0}$. The results in Theorem 4.1 enable us to prove the following

THEOREM 4.2. Let a linear connexion on $M$ be such that $T=0, \nabla R=W \otimes R$ and $W \not \equiv 0$, and let $M_{0}$ be the subset $\{u \in M: W(u)=0\}$. Then there exists on $M \backslash M_{0}$ some tensor $S$ of type $(1,2)$ which is

(a) symmetric in the two covariant indices,

(b) nowhere zero in $M \backslash M_{0}$, and

(c) such that on every coordinate neighborhood $U$ in $M \backslash M_{0}, R$ can be decomposed into

$$
R_{k j i}^{h}=W_{k} S_{j i}^{h}-W_{j} S_{k i}^{h} .
$$

Any two such tensors $S$ on $M \backslash M_{0}$ differ from each other by a tensor of the form $W \otimes W \otimes C$, where $C$ is some vector on $M \backslash M_{0}$.

Proof. Consider the bundle $B^{S}$ of all tensors $S$ of type $(1,2)$ at all points of $M \backslash M_{0}$ having the properties of the tensor $S_{j i}{ }^{h}$ in Theorem 4.1. Because the most general $S$ of such tensors at a point $u \in M \backslash M_{0}$ is of the form (4.2), the fiber $F_{u}$ of $B^{S}$ over $u$ is a linear space isomorphic to $R^{n}$, and is therefore solid. Then, by a theorem of Steenrod's $[6$, p. 55] on existence of cross sections on fiber bundles, $C^{\infty}$ cross sections of $B^{S}$ exist. Any such cross section is a tensor $S$ of type $(1,2)$ on $M \backslash M_{0}$ satisfying the conditions stated in the theorem. The last assertion in the theorem follows from (4.2), and this completes the proof.

4.3. Another local decomposition of the curvature tensor in $M \backslash M_{0}$.

THEOREM 4.3. Let a linear connexion on $M$ be such that $T=0, \nabla R=W \otimes R$ and $W \neq \equiv$, and let $M_{0}=\{u \in M: W(u)=0\}$. If the dimension of the holonomy group is $r(1 \leqq r \leqq n-1)$, then for each point $u$ of $M \backslash M_{0}$, there exists some coordinate neighborhood $U \ni u$ on which the curvature tensor can be decomposed into

$$
R_{k j i}{ }^{h}=\left(W_{k} W_{j}^{A}-W_{j} W_{k}^{A}\right)\left(W_{i} Z_{A}^{h}+W_{i}^{B} Z_{A B}^{h}\right) \quad(2 \leqq A, B \leqq r+1),
$$


where $W_{k}^{A}, Z_{A}^{h}, Z_{A B}^{h}=Z_{B A}^{h}$ are covectors and vectors on $U$ such that the $r+1$ covectors $W_{k}, W_{k}^{A}$ are everywhere linearly independent.

Proof. Comparing the two expressions

$$
\begin{aligned}
R_{k j i}{ }^{h} & =P_{k j}^{A} Q_{A i}{ }^{h}, \\
R_{k j i}{ }^{h} & =W_{k} S_{j i}{ }^{h}-W_{j} S_{k i}{ }^{h},
\end{aligned}
$$

for $R$ on $U$, we have

$$
W_{k i} S_{j i}{ }^{h}-W_{j i} S_{k i}{ }^{h}=P_{k j}^{A} Q_{A i}{ }^{h}
$$

Since $W$ has no zero in $U$, there exists on $U$ some vector $X^{k}$ such that $W_{k} X^{k}$ $=1$. Contraction of the above equation by $X^{k}$ gives

$$
S_{j i}{ }^{h}=\left(P_{k j}^{A} X^{k}\right) Q_{A i}{ }^{h}+W_{j}\left(S_{k i}{ }^{h} X^{k}\right)
$$

which we write as

$$
S_{j i}{ }^{h}=W_{j}^{A} Q_{A i}{ }^{h}+W_{j} Q_{i}{ }^{h} .
$$

Using this in (4.1), we obtain

$$
R_{k j i}{ }^{h}=\left(W_{k} W_{j}^{A}-W_{j} W_{k}^{A}\right) Q_{A i}{ }^{h} .
$$

The $r$ tensors $Q_{A i}{ }^{h}$ being independent, comparison of (2.2) with (4.7) gives

$$
P_{k j}^{A}=W_{k} W_{j}^{A}-W_{j} W_{k}^{A}
$$

Next, we show that the $r+1$ covectors $W_{k}, W_{k}^{A}$ are everywhere (in $U$ ) independent. Assume that this is not the case, i.e., at some point $u$ of $U$, $W_{k}(u)$ and $W_{\boldsymbol{k}}^{A}(u)$ are not independent. Then there exist some constants $c$ and $c_{A}$, not all zero, such that

$$
c W_{k}(u)+c_{A} W_{k}^{A}(u)=0,
$$

where, since $W(u) \neq 0$, the $c_{A}$ are not all zero. On account of this, we have from (4.8) that

$$
\begin{aligned}
c_{A} P_{k j}^{A}(u) & =W_{k}(u) c_{A} W_{j}^{A}(u)-W_{j}(u) c_{A} W_{k}^{A}(u) \\
& =-W_{k}(u) c W_{j}(u)+W_{j}(u) c W_{k}(u)=0 .
\end{aligned}
$$

But this contradicts the fact the $r$ tensors $P_{k j}^{A}$ are everywhere independent. Hence the $r+1$ vectors $W_{k}, W_{k}^{A}$ are everywhere independent.

Lastly, from the symmetry of $S_{j i}{ }^{h}$ in $j, i$, and from (4.6) we obtain 


$$
W_{j}^{A} Q_{A i}{ }^{h}+W_{j} Q_{i}^{h}=W_{i}^{A} Q_{A j}{ }^{h}+W_{i} Q_{j}^{h}
$$

Since $W_{j}, W_{j}^{A}$ are everywhere independent, we can complete them to a coframe $W_{j}^{\alpha}(1 \leqq \alpha \leqq n)$. Let $W_{\alpha}^{j}$ be its dual frame. Then contraction of (4.9) by $W_{B}^{\prime}$ gives

$$
Q_{B i}{ }^{h}=W_{i}^{A}\left(W_{B}^{j} Q_{A j}{ }^{h}\right)+W_{i}\left(W_{B}^{j} Q_{j}^{h}\right)
$$

We write this as

$$
Q_{A i}{ }^{h}=W_{i} Z_{A}^{h}+W_{i}^{B} Z_{A B}^{h}
$$

and substitute it in (4.9). The result is

$$
W_{i}\left(W_{j}^{A} Z_{A}^{h}-Q_{j}^{h}\right)-W_{j}\left(W_{i}^{A} Z_{A}^{h}-Q_{i}^{h}\right)=W_{j}^{B} W_{i}^{A}\left(Z_{A B}^{h}-Z_{B A}^{h}\right)
$$

Contracting this by $W_{1}^{j}$, we get

$$
Q_{i}^{h}=W_{i} Z^{h}+W_{i}^{A} Z_{A}^{h}, \text { where } Z^{h}=W_{1}^{j} Q_{j}^{h} .
$$

Consequently (4.11) reduces to $Z_{A B}^{h}=Z_{B A}^{h}$. This and (4.10) and (4.7) complete the proof of our theorem.

An easy consequence of (4.5) and Theorem $1.4(\mathrm{~d})$ is

THEOREM 4.4. The covectors and vectors $W_{k}^{A}, Z_{A}^{h}, Z_{A B}^{h}$ which appear in Theorem 4.3 are locally defined. If $U, U^{*}$ are any two intersecting coordinate neighborhoods on each of which there is a decomposition of $R$ of the form (4.5), then on $U \cap U^{*}$, we have

$$
\begin{aligned}
W_{k}^{* A^{*}} & =\phi_{A}^{A^{*}} W_{k}^{A}+\phi^{A^{*}} W_{k}, \\
Z_{A^{*}}^{* h} & =\phi_{A^{*}}^{A} Z_{A}^{h}-\phi^{B^{*}}{ }_{A^{*}}^{A} \phi_{B^{*}}^{B} Z_{A B}^{h}, \quad\left(2 \leqq A, B, A^{*}, B^{*} \leqq r+1\right) \\
Z_{A^{*} B^{*}}^{* h} & =\phi_{A^{*}}^{A} \phi_{B^{*}}^{B} Z_{A B}^{h}
\end{aligned}
$$

where $\phi^{A^{*}}, \phi_{A}^{A^{*}}, \phi_{A^{*}}^{A^{*}}$ are scalars on $U \cap U^{*}$ such that

$$
\phi_{A}^{A^{*}} \stackrel{B}{\phi_{A^{*}}^{*}}=\delta_{A}^{B}
$$

We now prove

THEOREM 4.5. The recurrence covector $W$ and the local covectors and vectors $W_{k}^{A}, Z_{A}^{h}, Z_{A B}^{h}$ which appear in

$$
R_{k j i}{ }^{h}=\left(W_{k} W_{j}^{A}-W_{j} W_{k}^{A}\right)\left(W_{i} Z_{A}^{h}+W_{i}^{B} Z_{A B}^{h}\right), \quad(2 \leqq A, B, C \leqq r+1)
$$

of Theorem 4.3 satisfy the following relations: 


$$
\begin{gathered}
r>1:\left\{\begin{array}{l}
\nabla_{l} W_{k}=N_{l} W_{k}, \\
\nabla_{l} W_{k}^{A}=N_{l B}^{A} W_{k}^{B}+N_{l}^{A} W_{k}, \\
\nabla_{l} Z_{A}^{h}=\tilde{L}_{l A}^{C} Z_{C}^{h}-N_{l} Z_{A}^{h}-N_{l}^{c} Z_{A C}^{h}, \\
\nabla_{l} Z_{A B}^{h}=\tilde{L}_{l A}^{C} Z_{C B}^{h}-N_{l B}^{c} Z_{A C .}^{h}
\end{array}\right. \\
r=1:\left\{\begin{array}{l}
\nabla_{l} W_{k}=N_{l} W_{k}+N_{l 2} W_{k}^{2}, \\
\nabla_{l} W_{k}^{2}=N_{l}^{2} W_{k}+N_{l 2}^{2} W_{k}^{2}, \\
\nabla_{l} Z_{2}^{h}=-N_{l}^{2} Z_{22}^{h}+\left(L_{l 2}^{2}-N_{l}\right) Z_{2}^{h}, \\
\nabla_{l} Z_{22}^{h}=\left(\tilde{L}_{l 2}^{2}-N_{l 2}^{2}\right) Z_{22}^{h}-N_{l 2} Z_{2}^{h} .
\end{array}\right.
\end{gathered}
$$

Proof. In Theorem 4.3 we have shown that for each point $u$ of $M \backslash M_{0}$, there exists some coordinate neighborhood $U \ni u$ on which

$$
R_{k j i}{ }^{n}=P_{k j}^{A} Q_{A i}{ }^{n} \quad(2 \leqq A, B \leqq r+1),
$$

where

$$
P_{k j}^{A}=W_{k} W_{j}^{A}-W_{j} W_{k}^{A}, \quad Q_{A i}{ }^{h}=W_{i} Z_{A}^{h}+W_{i}^{B} Z_{A B}^{h} .
$$

On the other hand, we have from Theorem 2.4 that

$$
\nabla_{l} P_{k j}^{A}=L_{l B}^{A} P_{k j}, \quad \nabla_{l} Q_{B i}{ }^{h}=\tilde{L}_{l B}^{A} Q_{A i}{ }^{h} .
$$

Substituting (4.15) in (4.16) $)_{1}$, we have

$$
\begin{aligned}
W_{k}\left(\nabla_{l} W_{j}^{A}\right) & +\left(\nabla_{l} W_{k}\right) W_{j}^{A}-W_{j}\left(\nabla_{l} W_{k}^{A}\right)-\left(\nabla_{l} W_{j}\right) W_{k}^{A} \\
& =L_{l B}^{A}\left(W_{k} W_{j}^{B}-W_{j} W_{k}^{B}\right) .
\end{aligned}
$$

Let us complete $W_{k}, W_{k}^{A}$ to a coframe $W_{k}^{\alpha}(1 \leqq \alpha \leqq n)$ and let $W_{\alpha}^{k}$ be its dual frame. Then contraction of (4.17) by $W_{1}^{k}$ and $W_{B}^{k}$ gives

$$
\begin{gathered}
\nabla_{l} W_{j}^{A}=\left[L_{l B}^{A}-\delta_{B}^{A}\left(\nabla_{l} W_{k}\right) W_{1}^{k}\right] W_{j}^{B}+\left(\nabla_{l} W_{k}^{A}\right) W_{1}^{k} W_{j}, \\
\left(\nabla_{l} W_{k}\right) W_{B}^{k} W_{j}^{A}-\left(\nabla_{l} W_{k}^{A}\right) W_{B}^{k} W_{j}-\delta_{B}^{A} \nabla_{l} W_{j}=-L_{l B}^{A} W_{j} .
\end{gathered}
$$

Substituting in the latter equation the expression (4.18) for $\nabla_{l} W_{k}^{A}$, we get

$$
\left(\nabla_{l} W_{k}\right) W_{B}^{k} W_{j}^{A}-\left[L_{l B}^{A}-\delta_{B}^{A}\left(\nabla_{l} W_{k}\right) W_{1}^{k}\right] W_{j}-\delta_{B}^{A} \nabla_{l} W_{j}=-L_{l B}^{A} W_{j},
$$

i.e.,

$$
\delta_{B}^{A}\left[\nabla_{l} W_{j}-\left(\nabla_{l} W_{k}\right) W_{1}^{k} W_{j}\right]=\left(\nabla_{l} W_{k}\right) W_{B}^{k} W_{j}^{A}
$$


It can be verified easily that (4.18) and (4.19) together are equivalent to (4.17) and thus to $(4.16)_{1}$.

Two cases arise according as $r>1$ or $r=1$.

Case 1. $r>1$. For $A \neq B,(4.19)$ gives

$$
\left(\nabla_{l} W_{j}\right) W_{B}^{j} W_{k}^{A}=0 .
$$

For any fixed $B$, choose an $A \neq B$. Since the covector $W^{A}$ has no zero, the above equation gives

$$
\left(\nabla_{l} W_{j}\right) W_{B}^{j}=0 \text {, }
$$

which is therefore true for every $B=2, \cdots, r+1$. On account of this, (4.19) reduces to

$$
\nabla_{l} W_{k}=\left(\nabla_{l} W_{j}\right) W_{1}^{j} W_{k} .
$$

We note that in this case $(r>1)$, the recurrence covector $W$ is recurrent.

Case 2. $r=1$. Then $A=B=2$, and (4.19) becomes

$$
\nabla_{l} W_{k}=\left(\nabla_{l} W_{j}\right) W_{1}^{j} W_{k}+\left(\nabla_{l} W_{j}\right) W_{2}^{j} W_{k}^{2}
$$

Now putting

$$
\left\{\begin{array}{l}
N_{l}=\left(\nabla_{l} W_{j}\right) W_{1}^{j}, \quad N_{l 2}=\left(\nabla_{l} W_{j}\right) W_{2}^{j}, \\
N_{l}^{A}=\left(\nabla_{l} W_{j}^{A}\right) W_{1}^{j}, \quad N_{l B}^{A}=L_{l B}^{A}-\delta_{B}^{A} N_{l}
\end{array}\right.
$$

in (4.20), (4.18) and (4.21), we obtain the first parts of (4.13) and (4.14) in the theorem.

Next, we consider equation (4.16) 2. For simplicity, let us put

$$
2 \leqq A, B \leqq r+1, \quad 1 \leqq \bar{A}, \bar{B}, \bar{C} \leqq r+1 .
$$

Then we have from $(4.15)_{2}$ and the first parts of (4.13) and (4.14) that

$$
Q_{A i}{ }^{h}=W_{i}^{\bar{B}} Z_{A \bar{B}}^{h}
$$

where $Z_{A B}^{h}=Z_{B A}^{h}, Z_{A 1}^{h}=Z_{A}^{h}$;

$$
\nabla_{l} W_{i}^{\bar{c}}=N_{i \bar{B}}^{\bar{c}} W_{i}^{\bar{B}},
$$

where $N_{l B}^{1}=0$ if $r>1$. On account of these, equation $(4.16)_{2}$, namely, $\nabla_{l} Q_{A i}{ }^{k}$ $=L_{l A}^{B} Q_{B i}{ }^{h}$ can be written

$$
\begin{aligned}
\tilde{L}_{l A}^{C} W_{i}^{\bar{B}} Z_{C \bar{B}}^{h} & =\nabla_{l}\left(W_{i \bar{B}}^{\bar{B}} Z_{A \bar{B}}^{h}\right)=\left(\nabla_{l} W_{i}^{\bar{B}}\right) Z_{A \bar{B}}^{h}+W_{i}^{\bar{B}} \nabla_{l} Z_{A \bar{B}}^{h} \\
& =N_{i \bar{C}}^{\bar{B}} W_{i}^{\bar{C}} Z_{A \bar{B}}^{h}+W_{i}^{\bar{B}} \nabla_{l} Z_{i \bar{B}}^{h}
\end{aligned}
$$


which is equivalent to

$$
\nabla_{1} Z_{A \bar{B}}^{h}=\widetilde{L}_{l A}^{C} Z_{C \bar{B}}^{h}-N_{l B}^{\bar{C}} Z_{A \bar{C}}^{h}
$$

This is the ccndensed form of the second parts of (4.13) and (4.14). Our theorem is thus completely proved.

\section{Certain parallel fields of coplanes and planes ON $M$ AND ON $M \backslash M_{0}$}

The decompositions of the curvature tensor into sums of tensor products in the last section give rise to the local fields of vectors $Z_{A}, Z_{A B}$ and covectors $W^{A}$ in $M \backslash M_{0}$. Here it will be shown that they (together with the recurrence covector $W$ ) span globally several parallel fields of planes or coplanes on $M \backslash M_{0}$. The pseudo-orthogonality between some of these parallel fields of planes and coplanes is closely related (i) to the rank of the Ricci tensor which will be proved to be always $\leqq 2$, and, (ii) in the case when the tensor $\nabla W$ is symmetric, to the condition for the holonomy group to be abelian.

5.1. The fields $D(W), D\left(W, W^{A}\right)$ of coplanes and the fields $D\left(Z_{A B}\right)$, $D\left(Z_{A}, Z_{A B}\right)$ of planes.

THEOREM 5.1. Let $M$ be a connected $n$-dimensional $C^{\infty}$-manifold with a linear connexion for which $T=0, \nabla R=W \otimes R, W \not \equiv$, and the holonomy group is of dimension $r$. Let $M_{0}$ be the subset $\{u \in M: W(u)=0\}$ and $M_{1}$ any connected component of $M \backslash M_{0}$, and let the curvature tensor be locally decomposed in $M \backslash M_{0}$ as

$$
R_{k j i}{ }^{h}=\left(W_{k} W_{j}^{A}-W_{j} W_{k}^{A}\right)\left(W_{i} Z_{A}^{h}+W_{i}^{B} Z_{A B}^{h}\right), \quad(2 \leqq A, B \leqq r+1) .
$$

Then

(a) On each $M_{1}$ the local fields of $(r+1)$-coplanes spanned by the covectors $W_{k}, W_{\mathbf{k}}^{A}$ piece together into a parallel field $D\left(W, W^{A}\right)$ of $(r+1)$-coplanes. The $k$-support of $R$ is a parallel field of $(r+1)$-coplanes on $M$ whose restriction to $M_{1}$ is $D\left(W, W^{A}\right)$.

(b) On each $M_{1}$ the local fields of planes spanned by the vectors $Z_{A}^{h}, Z_{A B}^{h}$ piece together into a parallel field $D\left(Z_{A}, Z_{A B}\right)$ of planes. The $h$-support of $R$ is a parallel field of planes on $M$ whose restriction to $M_{1}$ is $D\left(Z_{A}, Z_{A B}\right)$.

(c) On each $M_{1}$, the local fields of planes spanned by the vectors $Z_{A B}^{h}$ piece together into a field $D\left(Z_{A B}\right)$ of planes, but $D\left(Z_{A B}\right)$ is not necessarily of the same dimension everywhere in $M_{1}$.

(d) If the holonomy group is of dimension $r>1$, then

(i) $D(W)$ is a parallel field of 1-coplanes on $M \backslash M_{0}$.

(ii) On each $M_{1}$, the field $D\left(Z_{A B}\right)$ of planes defined in (c) is a parallel field of planes everywhere pseudo-orthogonal to the parallel field $D(W)$ of 1-coplanes. (We note here that in contrast with the other parallel fields described above, the 
parallel fields $D\left(Z_{\boldsymbol{A} B}\right)$ on different connected components of $M \backslash M_{0}$ may not be of the same dimension.)

Proof. In consequence of Theorem 2.2, to prove (a), it suffices to prove that at any point $u \in M \backslash M_{0}$, the $k$-support of $R$ coincides with the $(r+1)$ coplane spanned by the covectors $W_{k}, W_{k}^{A}$. Now let us complete $W_{k}, W_{k}^{A}$ to a coframe $W_{k}^{\alpha}$ and let $W_{\alpha}^{h}$ be its dual frame. Then the $k$-support of $R$ is spanned by the $n^{3}$ covectors:

$$
\begin{aligned}
{R_{k \alpha \mu}{ }^{\lambda}}^{\lambda} & =R_{k j i}{ }^{h} W_{\alpha}^{j} W_{\mu}^{i} W_{h}^{\lambda} \\
& =\left(W_{k} W_{j}^{A}-W_{j} W_{k}^{A}\right) W_{\alpha}^{j} Q_{A i}{ }^{h} W_{\mu}^{i} W_{h}^{\lambda} \\
& =\left(W_{k} \delta_{\alpha}{ }^{\lambda}-\delta_{\alpha}{ }^{1} W_{k}^{A}\right) Q_{A \mu}{ }^{\lambda} .
\end{aligned}
$$

Therefore, the $k$-support is contained in the $(r+1)$-coplane spanned by $W_{k}$, $W_{\boldsymbol{k}}^{A}$.

To show that the $k$-support contains all the $r+1$ covectors $W_{k}, W_{k}^{A}$, we first consider the covectors

$$
R_{k 1 \mu}^{\lambda}=-Q_{A \mu}{ }^{\lambda} W_{k}^{A} .
$$

The number of independent covectors among them is equal to the rank of the matrix $\left(Q_{A \mu}{ }^{\lambda}\right)$, where $A$ denotes the row and $(\mu, \lambda)$ the column. But this rank is equal to $r$ because the $r$ tensors $Q_{A i}{ }^{h}$ are independent. Therefore, the $k$-support of $R$ contains all the covectors $W_{\boldsymbol{k}}^{A}$.

Next consider the covectors

$$
R_{k 2 \mu}{ }^{\lambda}=Q_{2 \mu}{ }^{\lambda} W_{k} .
$$

Since the $r$ tensors $Q_{A i}^{h}$ are independent, at least one of the components $Q_{2 \mu}^{\lambda}$ is not zero. Therefore, the $k$-support of $R$ contains the covector $W_{k}$ also. This completes the proof of (a).

To prove (b), we use the same coframe $W_{\boldsymbol{k}}^{\alpha}$ as above, and obtain

$$
R_{\beta \alpha \mu}{ }^{h}=\left(\delta_{\beta}^{1} \delta_{\mu}^{A}-\delta_{\mu}^{1} \delta_{\beta}^{A}\right)\left(\delta_{\alpha}^{1} Z_{A}^{h}+\delta_{\alpha}^{B} Z_{A B}^{h}\right) .
$$

From this and

$$
R_{1 A 1}{ }^{h}=Z_{A}^{h}, \quad R_{1 A B}{ }^{h}=Z_{A B}^{h}
$$

it follows that the $h$-support of $R$ coincides with the plane spanned by the vectors $Z_{A}^{h}$ and $Z_{A B}^{h}$, and this proves (b).

(c) follows at once from $(4.12)_{3}$.

To prove (d), we observe that (i) and the first part of (ii) are consequences of (4.12), (4.13) and Theorem 1.1. It remains to prove that the parallel field 
$D\left(Z_{A B}\right)$ of planes is everywhere pseudo-orthogonal to the parallel field $D(W)$ of 1 -coplanes, i.e. to prove that

$$
W_{h} Z_{B C}^{h}=0 \text { if } \quad r>1 .
$$

For this purpose, let us substitute

$$
P_{k j}^{A}=W_{k} W_{j}^{A}-W_{j} W_{k}^{A}, \quad Q_{A i}{ }^{h}=W_{i} Z_{A}^{h}+W_{i}^{B} Z_{A B}^{h}
$$

into $(2.4)_{1}$, namely,

$$
P_{a[k}^{A} Q_{B j]}^{a}=C_{B C}^{A} P_{k j}^{c}
$$

Then we have

$$
\begin{aligned}
C_{B C}^{A}\left(W_{k} W_{j}^{C}-W_{j} W_{k}^{C}\right) & =\left(W_{a} W_{[k}^{A}-W_{[k} W_{a}^{A}\right)\left(W_{j]} Z_{B}^{a}+W_{j]}^{C} Z_{B C}^{a}\right) \\
= & \left(W_{a} Z_{B}^{a}\right)\left(W_{k}^{A} W_{j}-W_{j}^{A} W_{k}\right)-\left(W_{a}^{A} Z_{B C}^{a}\right)\left(W_{k} W_{j}^{C}-W_{j} W_{k}^{C}\right) \\
& +\left(W_{a} Z_{B C}^{a}\right)\left(W_{k}^{A} W_{j}^{C}-W_{j}^{A} W_{k}^{c}\right),
\end{aligned}
$$

i.e.,

$$
\left(C_{B C}^{A}+W_{a} Z_{B}^{a} \delta_{C}^{A}+W_{a}^{A} Z_{B C}^{a}\right)\left(W_{k} W_{j}^{C}-W_{j} W_{k}^{C}\right)=\left(W_{a} Z_{B C}^{a}\right)\left(W_{k}^{A} W_{j}^{C}-W_{j}^{A} W_{k}^{C}\right) .
$$

Comparison of the coefficient of $W_{k}$ shows that the left side is zero, and so also is the right side. From this it follows that $W_{a} Z_{B C}^{a}=0$ if $r>1$, as was to be proved. Note that we have also proved that

$$
C_{B C}^{A}+W_{a} Z_{B}^{a} \delta_{C}^{A}+W_{a}^{A} Z_{B C}^{a}=0 \text { if } r>1 .
$$

\subsection{Rank of the Ricci tensor.}

THEOREM 5.2. For a linear connexion with $T=0, \nabla R=W \otimes R$ and $W \not \equiv 0$, the Ricci tensor is of constant rank $\leqq 2$. The Ricci tensor is of rank 0 (i.e., $R_{j i}$ is everywhere zero) if the parallel fields $D\left(W, W^{A}\right), D\left(Z_{A}, Z_{A B}\right)$ are pseudoorthogonal; it is of rank $\leqq 1$ if the field $D(W)$ of 1 -cotatanes and the parallel field of planes $D\left(Z_{A}, Z_{A B}\right)$ are pseudo-orthogonal, or if the-pärallel field $D\left(W, W^{A}\right)$ of $(r+1)$-coplanes and the field $D\left(Z_{A B}\right)$ of planes are psendo-orthogonal.

Proof. We have proved in Theorem 2.1 that the rank of $R_{j i}$ is constant on $M$. Now we deduce from the local decomposition (4.5) of $R$ that

$$
\begin{aligned}
R_{j i}=R_{h j i}{ }^{h}= & \left(W_{h} Z_{A B}^{h}\right) W_{j}^{A} W_{i}^{B}+\left(W_{h} Z_{A}^{h}\right) W_{j}^{A} W_{i} \\
& -\left(W_{h}^{A} Z_{A B}^{h}\right) W_{j} W_{i}^{B}-\left(W_{h}^{A} Z_{A}^{h}\right) W_{j} W_{i} .
\end{aligned}
$$

It follows from this that the rank of $R_{j i}$ is equal to the rank of the $(r+1)$ $X(r+1)$ matrix 


$$
\left[\begin{array}{cr}
W_{h} Z_{A B}^{h} & W_{h} Z_{A}^{h} \\
-W_{h}^{A} Z_{A B}^{h} & -W_{h}^{A} Z_{A}^{h}
\end{array}\right] .
$$

If $r=1$, then $R_{j i}$ is obviously of rank $\leqq 2$. If $r>1$, then by (5.1) $W_{h} Z_{A B}^{h}=0$. Therefore, it is seen from (5.3) that in this case also, $R_{j i}$ is of rank $\leqq 2$. The remaining part of this theorem is an immediate consequence of (5.3).

5.3. A theorem on the holonomy group when $\nabla W$ is symmetric. It is known (Walker $[8, \S 6]$ ) that for a Riemannian connexion with $\nabla R=W \otimes R$ and $W \neq 0$, the tensor $\nabla W$ is symmetric. On the other hand Hlavaty [2, Theorem 3.3] has recently proved that the (local) holonomy group of such a Riemannian connexion is abelian. We now prove

THEOREM 5.3. For a linear connexion with $T=0, \nabla R=W \otimes R, W \not \equiv 0$ and $\nabla W$ symmetric, the holonomy group $H$ is abelian iff

(a) $\operatorname{dim} H=1$, or

(b) $\operatorname{dim} H>1$, the parallel fields $D(W)$ and $D\left(Z_{A}, Z_{A B}\right)$ are pseudoorthogonal, and the parallel fields $D\left(W, W^{A}\right)$ and $D\left(Z_{A B}\right)$ are pseudo-orthogonal.

Proof. If $\operatorname{dim} H=r=1, H$ is of course abelian.

Let us assume then $\operatorname{dim} H=r>1$. We have from (2.4) that

$$
\begin{aligned}
Q_{[B a}{ }^{h} Q_{C] i}{ }^{a} & =\widetilde{C}_{B C}^{A} Q_{A i}{ }^{h}, \\
\left(\nabla_{[m} W_{l]}\right) \delta_{C}^{A} & =\left(C_{B C}^{A}+\widetilde{C}_{B C}^{A}\right) P_{m l}^{B},
\end{aligned}
$$

where $2 \leqq A, B, C \leqq r+1$. Equation (5.4) shows that the condition for $H$ to be abelian is that $\widetilde{C}_{B C}^{A}=0$ (see corollary to Theorem 2.4 ). Since $\nabla W$ is symmetric and the $r$ tensors $P_{m l}^{C}$ are independent, it follows from (5.5) that $\widetilde{C}_{B C}^{A}=-C_{B C}^{A}$. Therefore, the condition for $H$ to be abelian is $C_{B C}^{A}=0$, i.e., by (5.2)

$$
W_{a} Z_{B C C}^{a}+W_{a}^{A} Z_{B C}^{a}=0, \quad(2 \leqq A, B, C \leqq r+1 ; r>1) .
$$

We now show that (5.6) is equivalent to

$$
W_{a} Z_{B}^{a}=0, \quad W_{a}^{A} Z_{B C}^{a}=0 .
$$

Since $Z_{B C}^{a}=Z_{C B}^{a}$, equation (5.6) implies that

$$
W_{a} Z_{B}^{a} \delta_{C}^{A}=W_{a} Z_{C}^{a} \delta_{B}^{A}
$$

If in this equation we let $B$ be arbitrary but fixed and $A=C \neq B$, we obtain $W_{a} Z_{B}^{a}=0$. Therefore, $(5.7)_{1}$ is true and, on account of this, (5.6) reduces to $(5.7)_{2}$. Our theorem now follows from (5.7) and (5.1): $W_{h} Z_{B C}^{h}=0$ which holds for $r>1$. 


\section{DECOMPOSITIONS OF THE TENSOR $\nabla W$}

In Theorem 4.5 we proved that for each point $u$ of $M$ at which $W(u) \neq 0$, there exists a coordinate neighborhood $U \ni u$ on which $\nabla W$ can be decomposed into

$$
\nabla_{l} W_{k}=N_{l} W_{k} \quad \text { if } r>1,
$$

or

$$
\nabla_{l} W_{k}=N_{l} W_{k}+\tilde{N}_{l} \tilde{W}_{k} \quad \text { if } r=1,
$$

where $r$ is the dimension of the holonomy group, and $N_{l}, \tilde{N}_{l}\left(=N_{l 2}\right), \tilde{W}_{k}\left(=W_{k}^{2}\right)$ are covectors on $U$.

Since both the recurrence covector $W$ and its covariant derivative $\nabla W$ are globally defined on $M$ and therefore on $M \backslash M_{0}$, we naturally want to know whether there exist global covectors $N, \tilde{N}, \tilde{W}$ on $M \backslash M_{0}$ so that the above decomposition of $\nabla W$ holds globally on $M \backslash M_{0}$. The main purpose of this section is to study this problem.

6.1. The case $\operatorname{dim} H>1$.

THEOREM 6.1. Let $M$ be a connected $n$-dimensional $C^{\infty}$-manifold with a linear connexion for which $T=0, \nabla R=W \otimes R$ and $W \not \equiv 0$, and let $M_{0}$ be the subset $\{u \in M: W(u)=0\}$. If the holonomy group is of dimension $r>1$, then either

(a) $\nabla W=\rho W \otimes W$ everywhere on $M \backslash M_{0}$, or

(b) $\nabla W=N \otimes W$ and $\nabla N=(W-N) \otimes N+V \otimes W$ everywhere on $M \backslash M_{0}$, where the covectors $N$ and $V$ are uniquely determined and $N$ is nowhere dependent on $W$. It follows that in case (b), the covectors $W$ and $N$ span a parallel field $D(W, N)$ of 2-coplanes on $M \backslash M_{0}$.

Proof. Let $r>1$. We have proved in Theorem 4.5 that

$$
\nabla_{l} W_{k}=N_{l} W_{k} \quad \text { locally in } M \backslash M_{0} .
$$

Since the tensor $\nabla_{[l} W_{k]}$ on $M$ is either everywhere zero or nowhere zero in $M$ (cf. Theorem 3.1) and by (6.1), $\nabla_{[l} W_{k]}=N_{[l} W_{k]}$, we may conclude that the local covector $N_{l}$ in $M \backslash M_{0}$ is either everywhere dependent on $W_{l}$ or nowhere dependent on $W_{l}$.

In the first case we have $\nabla_{l} W_{k}=\rho W_{l} W_{k}$, and (a) is proved.

Consider further the second case where $N_{l}$ is nowhere dependent on $W_{l}$. The covector $N_{l}$ is uniquely determined by (6.1). For, if $N_{l}^{*}$ is another covector such that $\nabla_{l} W_{k}=N_{l}^{*} W_{k}$, then from $N_{l}^{*} W_{k}=\nabla_{l} W_{k}=N_{l} W_{k}$, we obtain $\left(N_{l}^{*}-N_{l}\right) W_{k}=0$. Since $W$ is nowhere zero in $M \backslash M_{0}$, this is equivalent to $N_{l}^{*}=N_{l}$. In other words, we have a unique covector $N$ on $M \backslash M_{0}$ such that $\nabla W=N \otimes W$ everywhere in $M \backslash M_{0}$.

Substituting $\nabla_{l} W_{k}=N_{l} W_{k}$ in $\nabla_{m}\left(\nabla_{l l} W_{k]}\right)=W_{m} \nabla_{[l} W_{k]}$ (cf. Theorem 3.1), we obtain 


$$
\begin{aligned}
W_{m} N_{[l} W_{k]} & =\nabla_{m}\left(N_{[l} W_{k]}\right)=\left(\nabla_{m} N_{[l}\right) W_{k]}+N_{[l} \nabla_{m} W_{k]} \\
& =\left(\nabla_{m} N_{[l}\right) W_{k]}+N_{[l} N_{m} W_{k]} .
\end{aligned}
$$

Therefore,

$$
\left[\nabla_{m} N_{[l}-\left(W_{m}-N_{m}\right) N_{[l}\right] W_{k]}=0
$$

Hence

$$
\nabla_{m} N_{l}=\left(W_{m}-N_{m}\right) N_{l}+V_{m} W_{l},
$$

where $V_{m}$ is some covector on $U$. It follows from this (by the argument used above on $N_{l}$ ) that there exists on $M \backslash M_{0}$ a unique covector $V$ such that

$$
\nabla N=(W-N) \otimes N+V \otimes W .
$$

Combining this equation with $\nabla W=N \otimes W$ and the fact that $N$ is nowhere dependent on $W$, we see (cf. Theorem 1.1) that the covectors $W, N$ span a parallel field of 2-coplanes on $M \backslash M_{0}$. This completes the proof of the theorem.

6.2. The case $\operatorname{dim} H=1$. In this case, the tensors $R$ and $\nabla W$ can be locally decomposed in $M \backslash M_{0}$ as

$$
\begin{aligned}
R_{k j i}{ }^{h} & =\left(W_{k} \tilde{W}_{j}-W_{j} \tilde{W}_{k}\right)\left(W_{i} Z^{h}+\tilde{W}_{i} \tilde{Z}^{h}\right), \\
\nabla_{l} W_{k} & =N_{l} W_{k}+\tilde{N}_{l} \tilde{W}_{k} .
\end{aligned}
$$

Furthermore, the field $D(W, \tilde{W})$ of 2-coplanes on $M \backslash M_{0}$ is the restriction to $M \backslash M_{0}$ of the $k$-support of $R$. We now prove

TheOREM 6.2. If $\operatorname{dim} H=1$, the local covectors $N_{l}, \tilde{N}_{l}$ in $M \backslash M_{0}$ which appear in (6.2) have the following properties:

(a) $\tilde{N}_{l}$ is everywhere lying in the $k$-support of $R$.

(b) $N_{l}$ is either everywhere or nowhere lying in the $k$-support of $R$.

(c) If $N_{l}$ is nowhere lying in the k-support of $R$, then $D(N, W, \tilde{W})$ is a parallel field of 3-coplanes on $M \backslash M_{0}$.

Proof. Substituting (6.2) in (3.2), namely, $\operatorname{cyc}\left(W_{m} \nabla_{[l} W_{k]}\right)=0$, we have

$$
\operatorname{cyc} W_{m}\left(N_{[l} W_{k]}+\tilde{N}_{[l} \tilde{W}_{k]}\right)=0, \quad \text { i.e. } \quad \operatorname{cyc}\left(W_{m} \tilde{N}_{[l} \tilde{W}_{k]}\right)=0 .
$$

From this, since $W_{l}$ and $\tilde{W}_{l}$ are everywhere independent, it follows that

$$
\tilde{N}_{l}=\rho W_{l}+\tilde{\rho} \tilde{W}_{l},
$$

which proves (a).

On account of (6.3), we have

$$
\nabla_{[l} W_{k]}=N_{[l} W_{k]}+\tilde{N}_{[l} \tilde{W}_{k]}=N_{[l} W_{k]}-\rho \tilde{W}_{[l} W_{k]}=\left(N_{[l}-\rho \tilde{W}_{[l}\right) W_{k]} \text {, }
$$

i.e.

$$
\nabla_{[l} W_{k]}=\bar{N}_{l l} W_{k]}, \quad \text { where } \quad \bar{N}_{l}=N_{l}-\rho \tilde{W}_{l} .
$$


Combining this with (3.1), namely,

$$
\nabla_{m}\left(\nabla_{[l} W_{k]}\right)=W_{m} \nabla_{[l} W_{k]},
$$

we see that $\bar{N}_{[l} W_{k]}$ is everywhere zero or nowhere zero; in other words, either $N_{l}$ is everywhere dependent on $W_{l}$ and $\tilde{W}_{l}$ or nowhere dependent on $W_{l}$ and $\tilde{W}_{l}$. This proves (b).

Consider further the case where $\bar{N}_{[l} W_{k]}$ is nowhere zero. Substituting (6.4) in (6.5), we get

$$
W_{m} \bar{N}_{[l} W_{k]}=\nabla_{m}\left(\bar{N}_{[l} W_{k]}\right)=\left(\nabla_{m} \bar{N}_{[l}\right) W_{k]}+\bar{N}_{[l}\left(N_{m} W_{k]}+\tilde{N}_{m} \tilde{W}_{k]}\right)
$$

i.e.

$$
\left[\nabla_{m} \bar{N}_{[l}-\left(W_{m}-N_{m}\right) \bar{N}_{[l}\right] W_{k]}+\tilde{N}_{m} \bar{N}_{[l} \tilde{W}_{k]}=0 .
$$

Applying Cartan's lemma to this equation, we see that $\nabla_{m} \bar{N}_{l}$ is of the form

$$
\nabla_{m} \bar{N}_{l}=\left(W_{m}-N_{m}\right) \bar{N}_{l}+A_{m} W_{l}+\tilde{A}_{m} \tilde{W}_{l} .
$$

If we substitute $\bar{N}_{l}=N_{l}-\rho \tilde{W}_{l}$ in this, and take into account that $\nabla_{m} \tilde{W}_{l}$ $=N_{m}^{2} W_{l}+N_{m 2}^{2} \tilde{W}_{l}$ (cf. (4.14)), the result is of the form

$$
\nabla_{m} N_{l}=B_{m} N_{l}+C_{m} W_{l}+\tilde{C}_{m} \tilde{W}_{l} \text {. }
$$

On account of this and Theorem 1.1, (c) will be completely proved if we let $U, U^{*}$ be coordinate neighborhoods in $M \backslash M_{0}$ on which (6.2) and $\nabla_{l} W_{k}$ $=N_{l}^{*} W_{k}+\tilde{N}_{l}^{*} \tilde{W}_{k}^{*}$ respectively hold and are able to show that at every point of $U \cap U^{*}$, the covectors $W, \tilde{W}^{*}, N^{*}$ and the covectors $W, \tilde{W}, N$ span the same coplane. We already know that $\tilde{W}_{k}^{*}$ is linearly dependent on $W_{k}$ and $\tilde{W}_{k}$ (Theorem 4.4). In addition,

$$
N_{l}^{*} W_{k}+\tilde{N}_{l}^{*} \tilde{W}_{k}^{*}=\nabla_{l} W_{k}=N_{l} W_{k}+\tilde{N}_{l} \tilde{W}_{k}
$$

hold on $U \cap U^{*}$. Contracting this by a vector $X^{k}$ such that $W_{k} X^{k}=1$ and $\tilde{W}_{k}^{*} X^{k}=0$, we see that $N_{l}^{*}$ is linearly dependent on $N_{l}$ and $\tilde{N}_{l}$, and consequently, by (a), on $N_{l}, W_{l}$ and $\tilde{W}_{l}$. This completes the proof of the theorem.

The following theorem gives a partial answer to the question whether there exist global covectors $N, \tilde{N}, \tilde{W}$ on $M \backslash M_{0}$ such that equation (6.2) holds globally on $M \backslash M_{0}$.

Theorem 6.3. Let $\operatorname{dim} H=1$, and let $M_{00}$ be the subset

$$
\left\{u \in M:\left[\left(\nabla_{l} W_{[k}\right) W_{j]}\right](u)=0\right\}
$$

of $M \backslash M_{0}$. Then

(a) On $M_{00}$, there exists a unique covector $N$ such that

$$
\nabla W=N \otimes W .
$$

(b) On $\left(M \backslash M_{0}\right) \backslash M_{00}$, there exist 
(i) a covector $N$ which is an extension of the covector $N$ on $M_{00}$ defined in (a), and

(ii) a tensor $E$ of type $(0,2)$ and rank 1

such that

$$
\nabla W=N \otimes W+E .
$$

(c) If $E_{l k}$ are the components of $E$ in any local coordinate system in $\left(M \backslash M_{0}\right) \backslash M_{00}$, then at every point of $\left(M \backslash M_{0}\right) \backslash M_{00}$,

(i) both the $k$-support and the l-support of Elie in the $k$-support $(=D(W, \tilde{W}))$ of the curvature tensor $R$, and

(ii) the $k$-support of $E$ nowhere contains the recurrence covector $W$.

Proof. It follows from (6.2) that on a suitable coordinate neighborhood $U$ of each point in $M \backslash M_{0}$,

$$
\left(\nabla_{l} W_{[k}\right) W_{j]}=\tilde{N}_{\imath} \tilde{W}_{[k} W_{j]}
$$

Since $\tilde{W}_{[k} W_{j]}$ has no zero in $U$, the tensor $\left(\nabla_{l} W_{[k}\right) W_{j]}$ and the covector $\tilde{N}_{l}$ have common zeros in $U$. But $\left(\nabla_{l} W_{l k}\right) W_{j]}$ is a tensor on $M \backslash M_{0}$. Therefore, if $M_{00}$ is defined as in the theorem, we have $\nabla_{l} W_{k}=N_{l} W_{k}$ locally in $M_{00}$. Consequently (by an argument used in $\S 6.1$ ), there exists on $M_{00}$ a unique covector $N$ such that $\nabla W=N \otimes W$. This proves (a).

Let us now consider the situation on the submanifold $\left(M \backslash M_{0}\right) \backslash M_{00}$ on which $\nabla_{l} W_{k}$ can be locally expressed as

$$
\nabla_{l} W_{k}=N_{l} W_{k}+\tilde{N}_{l} \tilde{W}_{k},
$$

where $\tilde{N}_{l}$ has no zero. We recall that the vector $\tilde{W}_{k}$ was first introduced in the second local decomposition (\$4.3) of the curvature tensor $R$ in $M \backslash M_{0}$. Let $U$ be a coordinate neighborhood in $\left(M \backslash M_{0}\right) \backslash M_{00}$ on which $R$ can be decomposed as

$$
R_{k j i}{ }^{h}=\left(W_{k} \tilde{W}_{j}-W_{j} \tilde{W}_{k}\right)\left(W_{i} Z^{h}+\tilde{W}_{i} Z^{h}\right),
$$

where $\tilde{W}_{k}$ is everywhere independent of $W_{k}$. By Theorem 4.4, the most general covector $\tilde{W}_{k}^{*}$ which can appear in the second decomposition of $R$ is of the form

$$
\tilde{W}_{k}^{*}=\tilde{\phi} \tilde{W}_{k}+\phi W_{k}, \text { where } \tilde{\phi} \neq 0 .
$$

If at a point $u \in\left(M \backslash M_{0}\right) \backslash M_{00}, \nabla_{l} W_{k}$ can be expressed also as

$$
\nabla_{l} W_{k}=N_{l}^{*} W_{k}+\tilde{N}_{l}^{*} \tilde{W}_{k}^{*},
$$

then

$$
\begin{aligned}
N_{l} W_{k}+\tilde{N}_{l} \tilde{W}_{k} & =N_{l}^{*} W_{k}+\tilde{N}_{l}^{*} \tilde{W}_{k}^{*} \\
& =N_{l}^{*} W_{k}+\tilde{N}_{l}^{*}\left(\tilde{\phi} \tilde{W}_{k}+\phi W_{k}\right) \\
& =\left(N_{l}^{*}+\phi \tilde{N}_{l}^{*}\right) W_{k}+\tilde{\phi} \tilde{N}_{l}^{*} \tilde{W}_{k} .
\end{aligned}
$$


From this it follows that

$$
\begin{gathered}
\tilde{\phi} \tilde{N}_{l}^{*}=\tilde{N}_{l}, \quad \tilde{\phi} \neq 0, \\
N_{l}^{*}+\phi \tilde{N}_{l}^{*}=N_{l} .
\end{gathered}
$$

Therefore, the most general $N_{\imath}^{*}$ at $u$ satisfying $\left(6.2^{*}\right)$ is

$$
N_{l}^{*}=N_{l}-(\phi / \tilde{\phi}) \tilde{N}_{l} .
$$

Equations (6.7) and (6.8) show that on $\left(M \backslash M_{0}\right) \backslash M_{00}, \tilde{N}_{l}$ has a fixed direction and $N_{l}^{*}$ depends on a single parameter varying from $-\infty$ to $+\infty$.

Consider now the bundle $B^{N}$ of all covectors $N_{l}$ at all points of $\left(M \backslash M_{0}\right) \backslash M_{00}$. It follows from the above observations that the fiber $F_{u}$ over any point $u \in\left(M \backslash M_{0}\right) \backslash M_{00}$ is isomorphic with the real line. Therefore, by a theorem of Steenrod's on existence of cross sections on fiber bundles, $C^{\infty}$ cross sections of $B^{N}$ exist. Any such cross section is a vector $N^{*}$ on $\left(M \backslash M_{0}\right) \backslash M_{00}$ having the property that in a suitable coordinate neighborhood $U$ of each point of $\left(M \backslash M_{0}\right) \backslash M_{00}$,

$$
\nabla_{l} W_{k}=N_{l}^{*} W_{k}+\tilde{N}_{l} \tilde{W}_{k}, \quad \text { i.e. } \quad \nabla_{l} W_{k}-N_{l}^{*} W_{k}=\tilde{N}_{l} \tilde{W}_{k} .
$$

Since the left side of the last equation is the restriction to $U$ of a tensor on $\left(M \backslash M_{0}\right) \backslash M_{00}$ so is also the right side $\tilde{N}_{l} \tilde{W}_{k}$. In other words, there exists on $\left(M \backslash M_{0}\right) \backslash M_{00}$ a tensor $E$ of type $(0,2)$ and rank 1 such that $(E \mid U)_{l k}=\tilde{N}_{l} \tilde{W}_{k}$. We have thus shown that

$$
\begin{array}{ll}
\nabla W=N \otimes W & \text { on } M_{00}, \\
\nabla W=N^{*} \otimes W+E & \text { on }\left(M \backslash M_{0}\right) \backslash M_{00} .
\end{array}
$$

Let us now define the values of $N^{*}, E$ and $\tilde{N}$ on the boundary of $M_{00}$ by continuity. Then it is easily seen from (6.9) that

$$
E=0 \text { (i.e. } \tilde{N}=0 \text { ) and } N^{*}=N \text { on the boundary of } M_{00} \text {. }
$$

This completes the proof of (b).

Finally, (c) follows from the very definition of $E$ and from (6.7).

6.3. The special case where $\operatorname{dim} H=1$ and the Ricci tensor is not symmetric. For this case, we have a better result than Theorem 6.3.

Consider first the general case where $\operatorname{dim} H \geqq 1$. We have given the curvature tensor three different decompositions:

$$
\begin{aligned}
R_{k j i}{ }^{h} & =W_{k} S_{j i}{ }^{h}-W_{j} S_{k i}{ }^{h}, \\
R_{k j i}{ }^{2} & =P_{k j}{ }^{2}{ }_{A i}{ }^{h}, \\
R_{k j i}{ }^{h} & =\left(W_{k} W_{j}^{A}-W_{j} W_{k}^{A}\right)\left(W_{i} Z_{A}{ }^{h}+W_{i}^{B} Z_{A B}{ }^{h}\right), \quad(2 \leqq A, B \leqq r+1) .
\end{aligned}
$$

Of these, the first is global (on $M \backslash M_{0}$ ) while the other two are local (i.e. they hold on a suitable coordinate neighborhood $U$ of each point in $M \backslash M_{0}$ ). By (4.6), the global tensor $S_{j i}{ }^{h}$ can be expressed on $U$ as 


$$
S_{j i}{ }^{h}=W_{j}^{A} Q_{A i}{ }^{h}+W_{j} Q_{i}^{h}
$$

from which we have

$$
S_{j h}{ }^{h}=W_{j}^{A} Q_{A h}{ }^{h}+W_{j} Q_{h}^{h} .
$$

On the other hand, it follows from the equation

$$
-R_{[k j]}=R_{k j h}{ }^{h}=W_{k} S_{j h}{ }^{h}-W_{j} S_{k h}{ }^{h}
$$

that if the Ricci tensor is not symmetric, the global covector $S_{j h}{ }^{h}$ is everywhere (in $M \backslash M_{0}$ ) independent of $W_{j}$. Consequently the $r$ scalars $Q_{A h}{ }^{h}$ on $U$ which appear in (6.10) have no common zero. Hence, by (4.12) and (6.10), we may replace one of the $r$ covectors $W_{j}^{A}$ on $U$, say $W_{j}^{2}$, by the restriction of $S_{j h}{ }^{h}$ to $U$. Let us assume that this has been done. Then we have

$$
W_{j}^{2}=W_{j}^{A} Q_{A h}{ }^{h}+W_{j} Q_{h}{ }^{h} \text {, i.e. } Q_{A h}{ }^{h}=\delta_{A}{ }^{2}, \quad Q_{h}{ }^{h}=0
$$

Hence

LEMMA 6.1. If the Ricci tensor is not symmetric, we may take as $W_{\mathrm{j}}^{2}$ the restriction of $S_{j h}{ }^{h}$ to $U$ so that in the decomposition

$$
R_{k j i}{ }^{h}=\left(W_{k} W_{j}^{A}-W_{j} W_{k}^{A}\right)\left(W_{i} Z_{A}^{h}+W_{i}^{B} Z_{A B}^{h}\right), \quad(2 \leqq A, B \leqq r+1),
$$

the covector $W_{k}^{2}$, like $W_{k}$, is globally defined on $M \backslash M_{0}$, and $W_{h} Z_{A}^{h}+W_{h}^{B} Z_{A B}^{h}=\delta_{A}^{2}$.

Applying this lemma to the case where $\operatorname{dim} H=1$ and using some of the results in Theorems 5.1 and 6.2 , we have

THEOREM 6.4. Let a linear connexion on $M$ be such that $T=0, \nabla R=W \otimes R$ and $W \not \equiv 0$, and let $M_{0}=\{u \in M: W(u)=0\}$. If the holonomy group is of dimension 1 and the Ricci tensor is not symmetric, then the curvature tensor $R$ and the tensor $\nabla W$ can be globally decomposed on $M \backslash M_{0}$ as

$$
\begin{aligned}
R_{k j i}{ }^{h} & =\left(W_{k} \tilde{W}_{j}-W_{j} \tilde{W}_{k}\right)\left(W_{i} Z^{h}+\tilde{W}_{i} \tilde{Z}^{h}\right) \text { with } W_{h} Z^{h}+\tilde{W}_{h} \tilde{Z}^{h}=1, \\
\nabla W & =N \otimes W+\tilde{N} \otimes \tilde{W},
\end{aligned}
$$

where all the covectors and vectors are globally defined on $M \backslash M_{0}$ such that

(a) $D(\tilde{N}, W, \tilde{W})=D(W, \tilde{W})$ is a parallel field of 2-coplanes on $M \backslash M_{0}$,

(b) $D(N, W, \tilde{W})$ is either identical with $D(W, \tilde{W})$ or is a parallel field of 3-coplanes on $M \backslash M_{0}$,

(c) $D(Z, Z)$ is a parallel field of 1-planes or a parallel field of 2-planes on $M \backslash M_{0}$.

\section{REFERENCES}

1. V. Hlavaty, Holonomy group II. The Lie group induced by a tensor, J. Math. Mech. 8 (1959), 597-622. 
2. - Rigid motion in a Riemannian $V_{n}$. 1. A recurrent $V_{n}$, Rend. Circ. Mat. Palermo 9 (1960), 51-77.

3. K. Nomizu, Invariant affine connections on homogeneous spaces, Amer. J. Math. 76 (1954), 33-65. 1956.

4. - Lie groups and differential geometry, The Mathematical Society of Japan, Tokyo,

5. H. S. Ruse, $A$ classification of $K^{*}$-spaces, Proc. London Math. Soc. (2) 53 (1951), 212229.

6. N. E. Steenrod, The topology of fiber bundles, Princeton Univ. Press, Princeton, N. J., 1951.

7. A. G. Walker, On parallel fields of partially null vector spaces, Quart. J. Math. Oxford Ser. (2) 20 (1949), 135-145.

8. - On Ruse's spaces of recurrent curvature, Proc. London Math. Soc. (2) 52 (1950), 36-54.

9. Y. C. Wong, A class of non-Riemannian $K^{*}$-spaces, Proc. London Math. Soc. (3) 3 (1953), 118-128.

10. - Fields of parallel planes in affinely connected spaces, Quart. J. Math. Oxford Ser. (2) 4 (1953), 241-253.

11. - Subflat affinely connected spaces, Proceedings of the International Mathematical Congress, Amsterdam, 1954.

12. Y. C. Wong and K. Yano, Projectively flat spaces with recurrent curvature, Comment. Math. Helv. 35 (1961), 223-232.

13. Y. C. Wong, Recurrent tensors on a linearly connected differentiable manifold, Trans. Amer. Math. Soc. 99 (1961), 325-341.

14. - Two dimensional linear connexions with sero torsion and recurrent curvature (to appear).

UNIVERSITY OF HONG KONG,

HoNG Kong 